\title{
Reduced PRC2 function alters male germline epigenetic programming and paternal inheritance
}

\author{
Jessica M. Stringer ${ }^{1,2}$, Samuel C. Forster ${ }^{3,4,5}$, Zhipeng Qu ${ }^{6}$, Lexie Prokopuk ${ }^{1,5}$, Moira K. O’Bryan ${ }^{7}$, David K. Gardner ${ }^{8}$,
} Stefan J. White ${ }^{9}$, David Adelson ${ }^{6}$ and Patrick S. Western ${ }^{1,5^{*}}$ (i)

\begin{abstract}
Background: Defining the mechanisms that establish and regulate the transmission of epigenetic information from parent to offspring is critical for understanding disease heredity. Currently, the molecular pathways that regulate epigenetic information in the germline and its transmission to offspring are poorly understood.

Results: Here we provide evidence that Polycomb Repressive Complex 2 (PRC2) regulates paternal inheritance. Reduced PRC2 function in mice resulted in male sub-fertility and altered epigenetic and transcriptional control of retrotransposed elements in foetal male germ cells. Males with reduced PRC2 function produced offspring that over-expressed retrotransposed pseudogenes and had altered preimplantation embryo cleavage rates and cell cycle control.

Conclusion: This study reveals a novel role for the histone-modifying complex, PRC2, in paternal intergenerational transmission of epigenetic effects on offspring, with important implications for understanding disease inheritance.
\end{abstract}

Keywords: Germline, Epigenetic reprogramming, PRC2, H3K27me3, Paternal inheritance, Fertility

\section{Background}

Numerous studies have investigated the inheritance of physiological effects caused by environmental impacts on the parental genome, but the underlying epigenetic mechanisms regulating such inheritance are poorly understood $[1,2]$. It is well established that DNA methylation is passed through the germline (oocytes and sperm) to the following generation, where it influences gene activity, embryonic development and post-natal life $[1,3-5]$. In addition, recent studies have demonstrated effects of histone demethylases on inheritance [6, 7]. For example, zygotic over-expression of the Histone 3 lysine 27 (H3K27) demethylase, $K d m 6 b$, demonstrated a role for maternal H3K27 methylation in regulating DNA methylation-independent imprinting [7]. Similarly, increased levels of histone 3 lysine 4 dimethylation

\footnotetext{
* Correspondence: patrick.western@hudson.org.au

${ }^{1}$ Centre for Reproductive Health, Hudson Institute of Medical Research, Clayton, Victoria 3168, Australia

${ }^{5}$ Molecular and Translational Science, Monash University, Clayton, Victoria

3168, Australia

Full list of author information is available at the end of the article
}

(H3K4me2) in developing sperm resulted in paternally transmitted effects on health and development in mice [6]. In this study, we provide evidence that epigenetic inheritance in mice is also altered by a hypomorphic mutation in embryonic ectoderm development (Eed), a gene that is essential for H3K27 trimethylation (H3K27me3).

H3K27me3 is mediated by Polycomb Repressive Complex 2 (PRC2), which is comprised of the essential protein components EED, EZH2 and SUZ12 [8]. In mice, complete loss of function of any of these components results in loss of PRC2 activity, global reduction in H3K27me3 and embryonic lethality [9-12]. While complete loss of Eed results in lethality at gastrulation [13], germ cell-specific deletion results in male sterility [14]. However, an $N$-ethyl- $N$-nitrosourea (ENU)-induced hypomorphic allele, Eed ${ }^{l 7 R n 51989 S B}$, compromises PRC2 function and is compatible with survival, although some foetuses are lost during gestation due to defective placental development $[13,15]$. Eed $d^{l 7 R n 5-1989 S B}$ mice carry a point mutation at nucleotide 1989 that disrupts function of one 
of the WD repeat domains in the EED protein. This hypomorphic mutation does not abrogate the ability of EED to mediate H3K27 methylation as the Eed ${ }^{l 7 R n 5-1989 S B}$ allele can rescue H3K27 methylation in ES cells lacking the Eed gene [16]. Moreover, despite low EED function, adult mice with the hypomorphic Eed ${ }^{l 7 R n 5-1989 S B}$ mutation are fertile [17], allowing the investigation of PRC2 in epigenetic inheritance.

During embryonic development, epigenetic information is reprogrammed in the germline to ensure transmission of the correct information to the next generation. This involves extensive reorganisation of histone modifications and the removal of almost all DNA methylation from foetal germ cells [18-24]. In mice, removal of DNA methylation is initiated in migrating germ cells at around embryonic day (E)9, but is not complete until E13.5, after the germ cells have entered the developing gonads. Entry of germ cells into the gonads coincides with the removal of DNA methylation from imprinting control regions (ICRs), non-imprinted intergenic and intronic sequences and from many transposable elements (TEs), including LINE and SINE elements [18, 22-26]. During germline reprogramming, LINE and SINE elements are likely repressed by mechanisms other than DNA methylation to prevent TE expression and consequent insertional mutations $[18,26]$.

H3K27me3 broadly regulates developmental gene expression through its ability to repress target gene transcription. In foetal germ cells, H3K27me3 is enriched at developmental genes and on the $5^{\prime}$ flanking regions of some TEs, including LINE1 elements, intergenic regions, introns and imprint control regions [26-29]. Loss of function of the H3K9me3 methyltransferase SET domain Bifurcated 1 (SETDB1) in the developing male germline results in loss of DNA methylation, H3K9me3 and H3K27me3 at a subset of TEs [26]. This suggests that H3K27me3 functions with DNA methylation and H3K9me3 to co-regulate specific TEs in the germline [26]. Similarly, in cultured embryonic stem cells, H3K27me3 represses TEs in the absence of DNA methylation, establishing a functional requirement for H3K27me3 on these sequences [30].

H3K27me3 is enriched in foetal germ cells and in germ cells undergoing spermatogenesis [28, 29, 31, 32]. Moreover, H3K27me3 has been detected at developmental gene promoters in mature sperm, indicating that H3K27me3 may be transmitted to offspring and that such genes are poised for activation in the preimplantation embryo [33-36]. Another study showed retention of nucleosomes at repetitive sequences in sperm, including at LINE elements [37-39]. Together, these studies raise the possibility that $\mathrm{PRC} 2$ and $\mathrm{H} 3 \mathrm{~K} 27 \mathrm{me} 3$ regulate TEs during germline reprogramming and may modulate epigenetic inheritance in offspring. However, whether the potential inherited effects are directly mediated by histone modifications in offspring, or involve other mechanisms such as DNA methylation or altered inheritance of RNAs is unknown.

The aim of this study was to determine whether PRC2 contributes to the regulation of paternal epigenetic inheritance in a mammalian model. Using the hypomorphic Eed ${ }^{l 7 R n 5-1989 S B}$ mice, we provide evidence that PRC2 modulates H3K27me3 enrichment on TEs and represses retrotransposable LINE elements in the foetal male germline. Moreover, our data indicate that PRC2 is required in the paternal germline to regulate offspring development and repress a cohort of retrotransposed pseudogenes and related lincRNAs in offspring.

\section{Results}

Eed ${ }^{17 R n 5-1989 S B}$ mice are sub-fertile and provide a model for the study of epigenetic inheritance through the paternal germline

Since the primary aim of this study was to determine the role of EED in paternal epigenetic inheritance, we first assessed survival and male fertility in our colony of $E e d^{l 7 R n 5-1989 S B}$ mice. While the expected proportions of $E e d^{w t / w t}$ and $E e d^{w t / h y p o}$ offspring were produced, the

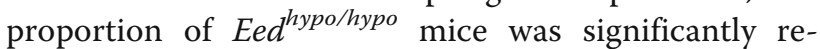
duced (ratio 1:2:0.1), demonstrating that Eed $d^{l 7 R n 5-1989 S B}$ homozygosity reduces viability (Additional file 1: Figure S1A). At E15.5 Eed $d^{w t / w t}, E e d^{w t / h y p o}$ and Eed $d^{\text {hypo/hypo }}$ foetuses were recovered in a 1:2:0.6 ratio (Additional file 1: Figure S1A). As few still-births or neonatal deaths were observed, we concluded that most Eed ${ }^{\text {hypo/hypo }}$ embryos died during the second half of gestation, consistent with previous observations $[13,17]$. Despite the loss of some foetuses, these experiments confirmed the survival of Eed ${ }^{\text {hypo/hypo }}$ males to adulthood, allowing the study of the male germline in a background of low EED function.

While previous studies found that homozygous Eed ${ }^{l 7 R n 5-1989 S B}$ mice produced offspring [17], the level of fertility in these mice remained unknown. We therefore completed a fertility analysis to determine whether the Eed hypomorphic mutation affected male germline function. Fertility was assessed in a cohort of hypomorphic Eed ${ }^{\text {hypo/hypo }}$ males $(n=13)$ compared to their age-matched Eed $d^{h y p o / w t}(n=13)$ and Eed ${ }^{w t / w t}(n=10)$ brothers, mated to wild-type females. Females were assessed for copulatory plugs as an indication of normal mating behaviour. Eed ${ }^{\text {hypo/hypo }}$ males sired $6.8 \pm 3.6$ pups per litter compared to $9.6 \pm 1.0$ and $8.7 \pm 1.7$ pups sired by $E e d^{h y p o / w t}$ and $E e d^{w t / w t}$ male controls, respectively. Notably, litters sired by Eed ${ }^{\text {hypo/hypo }}$ males were highly variable in size, resulting in a significantly increased standard deviation compared to Eed $d^{\text {hypo/wt }}$ and $E e d^{w t / w t}$ controls (Bartlett's test $p=0.0002$ ) (Fig. 1a). Eed hypo/hypo $^{\text {hing }}$ 


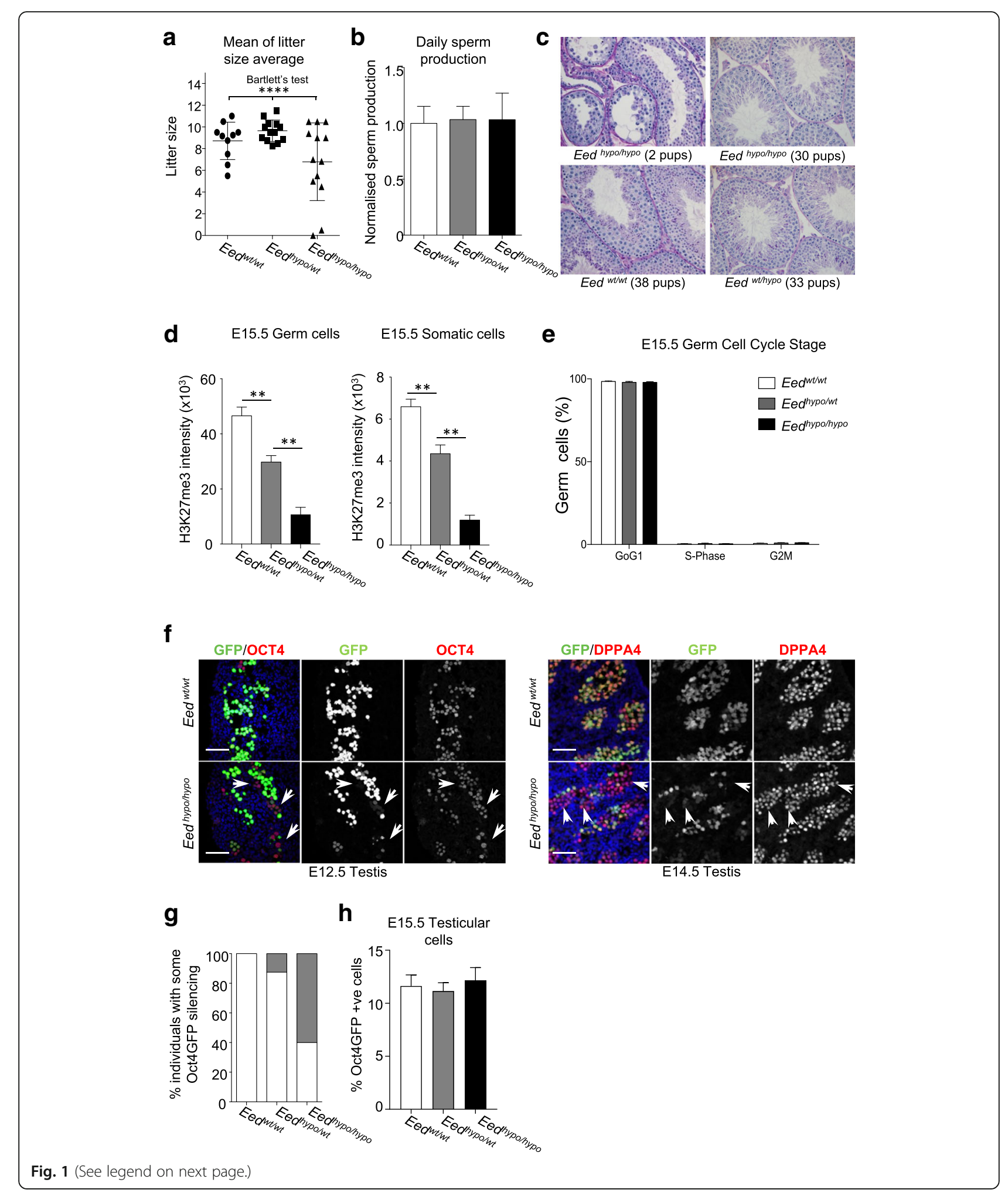


(See figure on previous page.)

Fig. 1 Reduced EED function resulted in male subfertility and reduced litter size. a Average litter size produced by wild-type female mice mated to Eed ${ }^{\text {wt }}$ wt $(n=10)$, Eed hypo/wt $(n=13)$ and Eed hypo/hypo $^{\prime}(n=13)$ males mated to for two periods of 30 days. Each point represents the average of two, four or six litters per male. Average litter sizes produced by Eed ${ }^{\text {hypo/hypo }}$ males were more variable in size than from Eed ${ }^{\text {wt } / \text { wt }}$ and Eed ${ }^{\text {hypo/wt }}$ males (Bartlett's test $P=0.0002$ ). b Normalised daily sperm production of Eed ${ }^{\text {wt }}$ wt $(n=3)$, Eed ${ }^{\text {hypo/wt }}(n=5)$, and Eed ${ }^{\text {hypo/hypo }}(n=6)$ males (mean \pm SEM, one-way ANOVA; no significant differences). c Testis histology from sub-fertile and fertile Eed ${ }^{\text {hypo/hypo }}$ males (top row) compared to their Eed ${ }^{w t}$ wt or Eed $^{\text {wthypo }}$ siblings (bottom images). In some Eed ${ }^{\text {hypo/hypo }}$ males (top left panel), testis cords contained reduced numbers of germ cells with evidence of germ cell sloughing, while other cords demonstrated apparently normal spermatogenesis. In some Eed ${ }^{\text {hypo/hypo }}$ males, testis cords appeared normal (top right panel). The total number of pups produced from the first four females is shown in parentheses. $\mathbf{d}$, e Flow cytometric analysis of H3K27me3 levels (d) and cell cycle stage (e) in germ and somatic cells of E15.5 Eed ${ }^{\text {wt }}{ }^{\text {twt }}$, Eed ${ }^{\text {wthypo }}$ and Eed ${ }^{\text {hypo/hypo }}$ testes. Eed $^{\text {wt }}$ wt $(n=5$ and 2), Eed ${ }^{\text {hypo/wt }}$ ( $n=7$ and 4 ) and Eed hypo/hypo ( $n=2$ and 3 ) testes in $\mathbf{d}$ and $\mathbf{e}$ respectively. Data are mean \pm SEM. $\mathbf{f}$ Immunofluorescent analysis of E12.5 and E14.5 Eed $d^{\text {hypo/hypo }}$ and Eed ${ }^{\text {wt }}$ wt foetal testis sections using antibodies specific for OCT4 at E12.5 and DPPA4 at E14.5. Merged images are shown in the left panels, with greyscale images of GFP shown in the middle panels and DPPA4 in the right-hand panels. Nuclear DNA is marked by DAPI in blue. Arrows indicate germ cells that lack Oct4GFP expression. Scale bar $50 \mu \mathrm{m}$. g Percentage of foetuses in which Oct4GFP silencing was observed in some germ cells at E12.5 and E14.5 in Eed ${ }^{\text {wt } / w t}(n=0 / 7)$, Eed ${ }^{\text {wt hypo }}(n=1 / 8)$ and Eed hypo/hypo $(n=6 / 10)$ animals. h Average percentage of cells in the total cell population that were Oct4GFP positive in pairs of E15.5 testes from Eed ${ }^{\text {wt } / w t}$ ( $n=13$ from 8 litters), Eed ${ }^{\text {wt/hypo }}$ ( $n=31$ form 17 litters) and Eed hypo/hypo ( $n=14$ from 9 litters) males (mean \pm SEM, One-way ANOVA; No significant differences)

males produced no pups or small litters more frequently than $E e d^{h y p o / w t}$ and $E e d^{w t / w t}$ males (Additional file 1: Figure S1B; chi-square $P=1.8 \mathrm{E}-05$ ), indicating sub-fertility in some Eed ${ }^{\text {hypo/hypo }}$ males. No difference was observed in the average daily sperm count between genotypes (Fig. 1b), and there was no correlation between litter size or frequency and male age (Additional file 1: Figure S1C).

Consistent with sporadic sub-fertility, testicular morphology of Eed ${ }^{\text {hypo/hypo }}$ males was also variable, but consistent with fertility outcomes. In obviously sub-fertile Eed ${ }^{\text {hypo/hypo }}$ males, germ cells were reduced and vacuoles present in some testis cords, indicating that germ cells were lost through sloughing (Fig. 1c). By contrast, normal testicular morphology was observed in Eed ${ }^{\text {hypo/hypo }}$ males that produced normal litter sizes (Fig. 1c). As a cohort $(n=20)$, abnormal testicular histology was only observed in four Eed ${ }^{\text {hypo/hypo }}$ males, with the remainder maintaining apparently normal testes with qualitatively normal spermatogenesis and weight. Combined, these data reflect the relatively mild sub-fertility and testicular phenotypes observed in these mice.

To determine whether the Eed hypomorphic mutation affected testis formation, we assessed the impact of reduced EED function on H3K27me3 levels and the development of germ and somatic cells in foetal testes. E12.5 and E15.5 were examined as they represent the earliest stages of testis formation and male germline development, and the completion of PRC2-dependent reorganisation of $\mathrm{H} 3 \mathrm{~K} 27 \mathrm{me} 3$ and initiation of DNA re-methylation in the paternal germline, respectively [1, 3-5, 40]. While H3K27me3 was detected in germ cells of all genotypes by immunofluorescence (Additional file 1: Figure S2A), flow cytometric assessment revealed significantly reduced global H3K27me3 levels in E15.5 germ and somatic cells in Eed ${ }^{\text {hypo/wt }}$ and Eed ${ }^{\text {hypo/hypo }}$ compared to Eed ${ }^{w t /}$ ${ }^{w t}$ testes (Fig. 1d, Additional file 1: Figure S2B). Reduced levels of H3K27me3 were presumably due to hypomorphic function of EED, as EED, EZH2 and SUZ12 were all detected in the germ and somatic cells of Eed $d^{w t / w t}, E e d^{h y p o / w t}$ and Eed ${ }^{h y p o / h y p o}$ testes (Additional file 1: Figure S2A).

Although H3K27me3 levels were reduced, the percentage of Sertoli and germ cells in the gonad (Additional file 1: Figure S2C-D), the proliferation of Sertoli cells (Additional file 1: Figure S2E) and the entry of germ cells into mitotic arrest (Fig. 1e) was unaffected. Similarly, qRTPCR analyses of a range of testis development genes involved in Sertoli, germ and steroidogenic cell development in E12.5 and E15.5 foetal testes (Additional file 1: Figure S2F), and flow cytometric analysis of SOX9 and AMH (Additional file 1: Figure S2G-H) revealed no differences between $E e d^{w t / w t} E e d^{h y p o / w t}$ and Eed ${ }^{\text {hypo/hypo }}$ testes. Collectively, these data demonstrated that reduced EED function significantly affected male fertility and germline H3K27me3 levels. However, the majority of males were able to produce litters in which epigenetic inheritance could be effectively studied.

\section{Reduced EED function resulted in stochastic silencing in male foetal germ cells}

To facilitate isolation of germ cells, our Eed hypomorphic mice carried a randomly integrated Oct4GFP transgene that is robustly transcribed in all foetal germ cells until birth, but remains silent in somatic cells [41, 42]. Initially, to confirm the veracity of the Oct4GFP transgene in this model, we used immunofluorescence to examine OCT4, DPPA4 and MVH in germ cells of E12.5 Eed ${ }^{w t / w t} \mathrm{Eed}^{\text {hypo }}$ ${ }^{w t}$ and Eed ${ }^{\text {hypo/hypo }}$ testes (Additional file 1: Figure S2I). Although OCT4, DPPA4 and MVH were detected in all germ cells, we observed silencing of Oct4GFP in some small patches of germ cells in some germ cells at E12.5 and E14.5 (Fig. 1f). However, this was not fully penetrant, as it affected $\sim 60 \%$ of Eed $d^{\text {hypo/hypo }}$ and $\sim 10 \%$ of Eed $d^{\text {wt } / \text { hypo }}$ individuals, and silencing was only evident in small numbers of germ cells (Fig. 1g). Indeed, analysis of FACS data 
revealed that there was no difference in the proportion of Oct4GFP-positive cells obtained from E15.5 foetal testes of Eed ${ }^{\text {hypo/hypo }}, E e d^{w t / h y p o}$ and Eed ${ }^{w t / w t}$ animals (Fig. 1h). This was consistent with similar numbers of MVH-positive germ cells in the testes of E15.5 Eed hypo/ $^{\text {hy }}$ hypo $E e d^{w t / h y p o}$ and $E e d^{w t / w t}$ animals (Additional file 1: Figure S2C). Combined, these data demonstrated that although normal numbers of foetal germ cells were present in Eed $d^{\text {hypo/hypo }}$ testes, occasional stochastic silencing of Oct4GFP occurred in germ cells of males with reduced EED function. As transgene silencing has been observed in other epigenetic models [43-45], we proposed that the stochastic Oct4GFP silencing in Eed ${ }^{\text {hypo/hypo }}$ germ cells was indicative of an altered epigenetic state in the germline of Eed hypomorphic males.

\section{H3K27me3 is required to repress LINE elements in male foetal germ cells}

To determine whether epigenetic state was disrupted in E15.5 male foetal germ cells we used ChIP-seq analysis to assess H3K27me3 enrichment in germ cells isolated from each of four single Eed $d^{\text {hypo/hypo }}$ male embryos and four $E e d^{w t / w t}$ male embryos. ChIP-seq yielded averages of 20,139,219 and 20,195,969 reads from the Eed hypo/hypo $^{\text {ho }}$ and $E e d^{w t / w t}$ samples, respectively, of which 97.2-97.9\% were alignable to the $\mathrm{mm} 10$ reference genome using bowtie2 (Additional file 1: Figure S3). HOMER analysis using a search region size of 1100 bp identified 60,933 and 55,453 H3K27me3 peaks in the Eed ${ }^{\text {hypo/hypo }}$ and $E e d^{w t / w t}$ samples, respectively (Additional file 2: Table S1 and Additional file 3: Table S2). Importantly, comparison of our data to three similar datasets demonstrated significant overlap at known PRC2 targets (Additional file 1: Figure S3G, Additional file 4: Table S3), demonstrating high specificity of the ChIP analysis. In addition, visualisation of normalised read counts in the Eed $d^{\text {hypo/hypo }}$ and Eed ${ }^{w t / w t}$ germ cell samples demonstrated clear H3K27me3 enrichment in the 5-prime regions of PRC2 target genes and no enrichment on a constitutively expressed gene $S d h a$, demonstrating sensitivity of the assay (Additional file 1: Figure S3H).

In addition, a high proportion of peaks were identified at repeat elements including LINE, LTR and SINE elements in both Eed ${ }^{\text {hypo/hypo }}$ and Eed ${ }^{w t / w t}$ germ cell samples (Fig. 2a). We used hypergeometric testing to determine whether the expected number of repeats was represented for each repeat category in the ChIP-seq data for $E e d^{w t / w t}$ and $E e d^{h y p o / h y p o}$ germ cells. In both $E e d^{\text {hypo/hypo }}$ and $E e d^{w t / w t}$ samples, SINE elements were substantially under-represented (fold enrichment $=1.31$ and 1.22, respectively; $p \sim 0$ ) in $\mathrm{H} 3 \mathrm{~K} 27 \mathrm{me} 3$-enriched peaks, but LINE elements were substantially over-represented (fold enrichment $=0.51$ and 0.61 , respectively; $P \sim 0$; Additional file 5: Table S4), suggesting that LINE elements were preferentially captured in the ChIP seq assay, but SINE elements were not (Additional file 5: Table S4). LTRs were represented at expected ratios in Eedhypo/hypo and Eed $d^{w t / w t}$ samples (fold enrichment $=1.04$ and 1.0, respectively; Additional file 5: Table S4). Together, these data indicated strong enrichment of H3K27me3 not only at PRC2 target loci, but also at some repeat sequences, most notably LINE elements.

To investigate locus-specific variation in H3K27me3, we used HOMER to identify specific sequences with differential H3K27me3 enrichment by comparing Eed ${ }^{\text {hypo/ }}$ hypo samples to $E e d^{w t / w t}$ samples (Additional file 1: Figure S3I-J). As H3K27me3 has previously been implicated in repression of both coding and non-coding sequences in foetal male germ cells [26, 28], we included repetitive sequences in our analyses. Samples were compared using $E e d^{w t / w t}$ as the baseline target and searching for regions with a cumulative Poisson $P$ value less than 0.0001 (sequencing-depth dependent) and $\geq 2$-fold reduction in H3K27me3 precipitated sequences. This revealed 923 regions with $\geq 2$-fold reduction in H3K27me3 in Eed $d^{\text {hypo/hypo }}$ compared $E e d^{\text {wt/wt }}$ germ cells (i.e. WT > HOM). The reciprocal comparison using Eed ${ }^{\text {hypo/hypo }}$ samples as baseline and $E e d^{w t / w t}$ samples as target identified 1,158 regions with $\geq 2$-fold increased H3K27me3 in Eed hypo/hypo germ cells (i.e. HOM > WT). Only 58 of these regions associated with coding genes ( 35 decreased and 23 increased, $2.84 \%$ of all differential peaks), while 1,951 LINE, LTR, SINE, intergenic and intronic sequences were identified with significantly different $(\geq$ 2-fold) levels of H3K27me3 (Fig. 2a; Additional file 6: Table S5 and Additional file 7: Table S6). These included 120 LINE, 93 LTR, 56 SINE, 378 intergenic and 206 intronic with decreased (Fig. 2a) and 362 LINE, 187 LTR, 52 SINE, 377 intergenic and 120 intronic with increased (Fig. 2a) H3K27me3 in Eed hypo/hypo compared to Eed $^{w t / w t}$ germ cells, representing $>97 \%$ of all differential ChIP peaks (Fig. 2b). Using a more stringent analysis employing edgeR, with a false discovery rate cut off of $P<0.05$, we identified 7 refGene annotated LINE1 loci with significantly fewer reads mapped in Eed ${ }^{\text {hypo/hypo }}$ compared to the $E e d^{w t / w t}$ samples (Additional file 8: Table S7), but no LINE1 elements with significantly more reads mapped in Eed $d^{\text {hypo/hypo }}$ compared to the Eed ${ }^{w t / w t}$ samples. Together, these data indicated that subtle differences in H3K27me3 regulation occurred predominantly at repetitive sequences, introns and intergenic regions in Eed hypo/hypo $^{\text {germ cells. }}$

We next examined the representation of each sequence category with differential H3K27me3 enrichment in the ChIP-seq data relative to the expected representation of annotated sequence classes across the genome. For repeats, hypergeometric testing was used to analyse the representation for each repeat category gaining or 
a Major sequence classes identified in $\mathrm{H} 3 \mathrm{~K} 27 \mathrm{me} 3$ enriched regions in Eed ${ }^{\text {hypo/hypo }}$ and Eed ${ }^{\text {wt/wt }}$ germ cells

\begin{tabular}{|c|c|c|c|c|c|}
\hline Category & 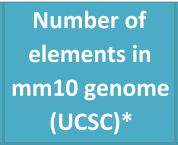 & $\begin{array}{c}\text { Number of peaks } \\
\text { annotated as } \\
\text { each category in } \\
\text { WT }\end{array}$ & $\begin{array}{c}\text { Number of peaks } \\
\text { annotated as } \\
\text { each category in } \\
\text { HOM }\end{array}$ & $\begin{array}{c}\text { Number of peaks } \\
\text { annotated as } \\
\text { each category in } \\
\text { WT > HOM }\end{array}$ & $\begin{array}{l}\text { Number of peaks } \\
\text { annotated as } \\
\text { each category in } \\
\text { HOM > WT }\end{array}$ \\
\hline LINE & 989,435 & 13,647 & 16,580 & 120 & 362 \\
\hline LTR & 973,534 & 7,094 & 8,764 & 93 & 187 \\
\hline SINE & $1,533,283$ & 2,510 & 2,517 & 56 & 52 \\
\hline Low comp & 386,539 & 303 & 317 & 8 & 8 \\
\hline intron & 296,183 & 7,529 & 7,259 & 206 & 120 \\
\hline Intergenic & 35,778 & 20,730 & 21,730 & 378 & 377 \\
\hline Total & $4,214,752$ & 51,813 & 57,167 & 861 & 1,106 \\
\hline
\end{tabular}

* Based on mm10 annotations in UCSC with LINE LTR and SINE defined by repeat masker.

b

H3K27me3: Eed ${ }^{\text {hypo/hypo }}<$ Eed ${ }^{\text {wt/wt }}$

Promoter (yellow) $\mathbf{0 . 7 5 \%}$

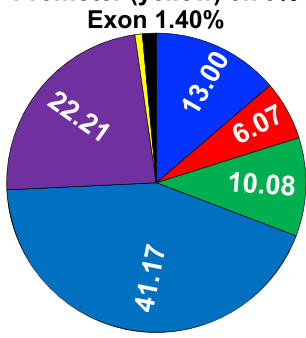

H3K27me3: Eed hypo/hypo $>$ Eed ${ }^{\text {wt/wt }}$

Promoter (yellow) $0.43 \%$
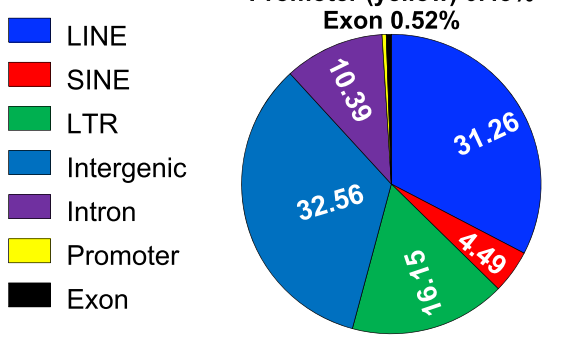

C i. Repeats at which H3K27me3 was reduced in Eed ${ }^{\text {hypo/hypo }}$ compared to Eed ${ }^{w t / w t}$ germ cells

\begin{tabular}{|c|c|c|c|c|c|c|c|c|c|}
\hline Category & $\begin{array}{l}\text { Genome } \\
\text { number }\end{array}$ & $\begin{array}{l}\text { Genome } \\
\text { total }\end{array}$ & $\begin{array}{l}\text { Genome } \\
\text { frequency }\end{array}$ & $\begin{array}{c}\text { WT }>\text { HOM } \\
\text { number }\end{array}$ & $\begin{array}{c}\text { WT }>\text { HOM } \\
\text { total }\end{array}$ & $\begin{array}{l}\text { WT > HOM } \\
\text { frequency }\end{array}$ & $\begin{array}{c}\text { Fold } \\
\text { enrichment }\end{array}$ & $\begin{array}{l}\text { P value (Under } \\
\text { representation) }\end{array}$ & $\begin{array}{l}\text { P value (Over } \\
\text { representation) }\end{array}$ \\
\hline LINE & 989435 & 5147736 & 0.19 & 266 & 1470 & 0.18 & 0.94 & 0.13 & 0.87 \\
\hline LTR & 973534 & 5147736 & 0.19 & 268 & 1470 & 0.18 & 0.96 & 0.24 & 0.76 \\
\hline SINE & 1533283 & 5147736 & 0.3 & 348 & 1470 & 0.24 & 0.79 & $6.99 \mathrm{E}-08$ & 1.00 \\
\hline Low Comp & 386539 & 5147736 & 0.08 & 150 & 1470 & 0.1 & 1.36 & 1.00 & 0.0001 \\
\hline
\end{tabular}

ii. Repeats at which H3K27me3 was greater in Eed ${ }^{\text {hypo/hypo }}$ compared to Eed ${ }^{\text {wtwt }}$ germ cells

\begin{tabular}{|c|c|c|c|c|c|c|c|c|c|}
\hline Category & $\begin{array}{l}\text { Genome } \\
\text { number }\end{array}$ & $\begin{array}{c}\text { Genome } \\
\text { total }\end{array}$ & $\begin{array}{l}\text { Genome } \\
\text { frequency }\end{array}$ & $\begin{array}{c}\text { HOM }>\text { WT } \\
\text { number }\end{array}$ & $\begin{array}{c}\text { HOM > WT } \\
\text { total }\end{array}$ & $\begin{array}{l}\text { HOM }>W T \\
\text { frequency }\end{array}$ & $\begin{array}{l}\text { Fold } \\
\text { enrichment }\end{array}$ & $\begin{array}{l}\text { P value (Under } \\
\text { representation) }\end{array}$ & $\begin{array}{l}\mathrm{P} \text { value (Over } \\
\text { representation) }\end{array}$ \\
\hline LINE & 989435 & 5147736 & 0.19 & 801 & 3005 & 0.27 & 1.39 & 1 & 0 \\
\hline LTR & 973534 & 5147736 & 0.19 & 617 & 3005 & 0.21 & 1.09 & 0.99 & 0.01 \\
\hline SINE & 1533283 & 5147736 & 0.3 & 372 & 3005 & 0.12 & 0.42 & 3.89E-114 & 1 \\
\hline Low Comp & 386539 & 5147736 & 0.08 & 340 & 3005 & 0.11 & 1.51 & 1 & 7.51E-14 \\
\hline
\end{tabular}

d

\section{H3K27me3 gain or loss at introns and intergenic regions}

\begin{tabular}{|c|c|c|c|c|c|c|c|}
\hline $\begin{array}{l}\text { H3K27me3 } \\
\text { gain / loss }\end{array}$ & Category & $\begin{array}{l}\text { Number of } \\
\text { peaks with } \\
\text { H3K27me3 } \\
\text { gain or loss }\end{array}$ & $\begin{array}{c}\text { Total } \\
\text { number of } \\
\text { H3K27me3 } \\
\text { peaks }\end{array}$ & $\begin{array}{l}\text { Expected } \\
\% \text { of peaks } \\
\text { identified }\end{array}$ & $\begin{array}{l}\text { Observed } \\
\% \text { of peaks } \\
\text { identified }\end{array}$ & P-value & $\begin{array}{c}\text { Represent } \\
\text { ation }\end{array}$ \\
\hline \multirow{2}{*}{$\begin{array}{l}H O M \\
>W T\end{array}$} & INTRON & 205 & 1158 & 22 & 22.21 & 0.96 & normal \\
\hline & INTERGENIC & 380 & 1158 & 30.93 & 41.17 & $<0.0001$ & over \\
\hline \multirow{2}{*}{$\begin{array}{l}W T> \\
H O M\end{array}$} & INTRON & 120 & 923 & 22 & 10.36 & $<0.0001$ & under \\
\hline & INTERGENIC & 377 & 923 & 30.93 & 32.56 & 0.42 & normal \\
\hline
\end{tabular}

e Transcript enrichment: Eed ${ }^{\text {hypo/hypo }}$ vs Eed'wt/wt
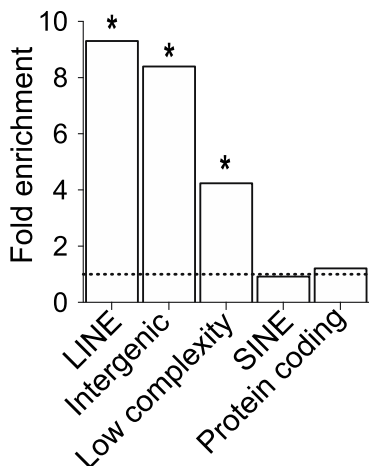

Fig. 2 (See legend on next page.) 
(See figure on previous page.)

Fig. 2 Reduced EED leads to epigenetic dysregulation of transposable elements in the paternal germline. a Major sequence classes identified with H3K27me3 enrichment identified by ChIP-seq in FACS purified E15.5 Eed ${ }^{\text {wt } / W t}(n=4)$ and Eed ${ }^{\text {hypo/hypo }}(n=4)$ germ cells. b Graphical summary of regions with loss or gain of H3K27me3 in Eed ${ }^{\text {hypo/hypo }}$ germ cells $(n=4)$ compared to Eed ${ }^{\text {wt } / w t}$ germ cells $(n=4)$. c Hypergeometric analysis of the expected and observed representation of sequences on which H3K27me3 was reduced (i) or increased (ii) in Eed ${ }^{\text {hypo/hypo }}(n=4)$ compared to Eed $^{\text {wt } / w t}(n=4)$ male E15.5 germ cells, grouped by annotation. $P$ values for over- and under-representation of each sequence category are shown, with $P<0.05$ considered significant. $\mathbf{d}$ Chi-square analysis of the expected and observed representation of sequences on which H3K27me3 was increased $(\mathrm{HOM}>\mathrm{WT})$ or reduced $(\mathrm{WT}>\mathrm{HOM})$ in Eed ${ }^{\text {hypo/hypo }}(n=4)$ compared to Eed ${ }^{\text {wt }} / \mathrm{wt}(n=4)$ male E15.5 germ cells, grouped by annotation. Intergenic sequences were significantly over-represented in sequences gaining H3K27me3, while intronic sequences were significantly underrepresented in sequences losing H3K27me3 in Eed ${ }^{\text {hypo/hypo }}$ germ cells. e Fold enrichment of transcripts from LINE, intergenic, low complexity, SINE and protein-coding genes in germ cells from E15.5 Eed ${ }^{\text {hypo/hypo }}(n=4)$ and Eed ${ }^{\text {wt } / w t}(n=4)$ foetuses. ${ }^{*} P<0.05$ (Fisher exact test). Dotted line illustrates no enrichment relative to random distribution

losing H3K27me3 in Eed ${ }^{w t / w t}$ and Eed $d^{\text {hypo/hypo }}$ germ cells relative to the total number of repeats in the genome. For example, LINE elements occupy $19 \%$ of all genome repeats (Fig. 2c (i)), and $18 \%$ of repeats were identified with reduced $\mathrm{H} 3 \mathrm{~K} 27 \mathrm{me} 3$ in Eed hypo/hypo $^{\text {compared to }}$ $E e d^{w t / w t}$ germ cells which were LINE elements (Fig. 2c (i)). In contrast, $27 \%$ of repeat sequences were identified

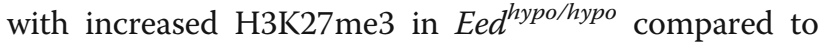
$E e d^{w t / w t}$ germ cells which were LINE elements, a significantly higher proportion than the expected 19\% (Fig. 2c (ii)). Thus, LINE elements with increased H3K27me3 were significantly over-represented in Eed $d^{\text {hypo/hypo }}$ germ cells (Fig. 2c; fold enrichment 1.39, $P \sim 0$ ), but LINE elements with reduced $\mathrm{H} 3 \mathrm{~K} 27 \mathrm{me} 3$ were detected at the expected frequency. LTRs were very moderately over-represented in peaks with increased H3K27me3 in Eed hypo/hypo germ cells (Fig. 2c; observed 21\%, expected $19 \%$; enrichment ratio $1.09 ; P=0.01)$. In contrast, SINE elements were under-represented in repeats with either reduced or increased $\mathrm{H} 3 \mathrm{~K} 27 \mathrm{me} 3$, a result that was consistent with overall under-representation of SINE elements in both the Eed $d^{\text {hypo/hypo }}$ and Eed ${ }^{w t / w t}$ germ cell H3K27me3 ChIPseq datasets (Fig. 2c). Although very few low complexity repeats had altered H3K27me3 in the ChIPseq dataset, these sequences were over-represented in both H3K27me3 gain and loss categories (i.e. some low complexity sequences gained H3K27me3, while others lost H3K27me3).

For non-repetitive genomic sequences (e.g. intergenic, intronic and promoters), we determined whether the expected percentage of peaks was represented for each sequence category in the ChIPseq data relative to the percentage of the total genome occupied by each sequence category. For example, intergenic sequences occupy 30.93\% of the genome, but were represented at normal frequency in peaks with decreased $(32.96 \%, P=0.42$, chi-square analysis) but were over-represented in peaks with increased H3K27me3 (41.17\%, $P<0.0001$; Fig. 2d). In contrast, intronic sequences were under-represented in peaks losing $\mathrm{H} 3 \mathrm{~K} 27 \mathrm{me} 3$ in Eed hypo/hypo $^{\text {germ cells }}$ but were normally represented in peaks gaining H3K27me3 (Fig. 2d). Promoters, exons, 5'UTRs, 3'
UTRs, small RNAs, tRNAs, rRNAs and CpG islands were not significantly over- or under-represented in peaks with either increased or decreased H3K27me3 in Eed hypo/hypo $^{\text {How }}$ compared to Eed ${ }^{w t / w t}$ germ cells. Together, these data indicated that H3K27me3 was redistributed throughout the genome of Eed ${ }^{\text {hypo/hypo }}$ compared to $\mathrm{Eed}^{w t / w t}$ germ cells. Although this apparently resulted in increased H3K27me3 at some intergenic and repeat sequences, significant numbers of LINE, LTR, SINE elements, intergenic and intronic sequences were detected with reduced H3K27me3.

Since H3K27me3 is a repressive modification, reduced H3K27me3 may result in increased transcription from the underlying sequence. To determine whether this was the case, RNA sequencing (RNA-seq) was performed to an average depth of $>20$ million reads per sample on FACS purified E15.5 male foetal germ cells using four independent Eed ${ }^{\text {hypo/ }}$ hypo and four $E e d^{w t / w t}$ samples (Additional file 1: Figure S4A). Comparison of 1000 genes that were not differentially expressed indicated a high level of technical consistency between the sample sets. (Additional file 1: Figure S4B). Similarly, principal component analysis revealed strong correlation between the RNA-seq sample sets generated from $\mathrm{Eed}^{w t / w t}$ germ cells (Additional file 1: Figure S4C), although, notably, there appeared to be greater variation be-

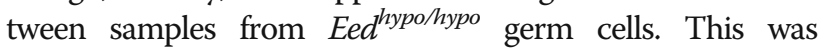
reminiscent of the observed stochastic variation in Oct4GFP

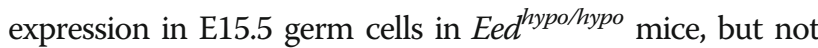
in $E e d^{w t / w t}$ mice. Consistent with the lack of differences in H3K27me3 enrichment at protein-coding genes, we observed no differences in expression of protein-coding genes in Eed $d^{\text {hypo/hypo }}$ compared to Eed ${ }^{w t / w t}$ germ cells using a significance limit of $P<0.01$ with Benjamini-Hochberg false detection correction. However, analysis of repetitive sequences annotated using HOMER, including TEs, revealed significant enrichment of RNA-seq reads mapping to annotated LINE elements $(P=0.033$, Fisher Exact Test, Benjamini-Hochberg false detection correction), intergenic $(P=0.0364$, Fisher Exact Test, Benjamini-Hochberg false detection correction) and low complexity sequences $(P=0.025$, Fisher Exact Test, Benjamini-Hochberg false detection correction) in Eed $d^{\text {hypo/hypo }}$ germ cells, although reads mapping to 
SINE sequences and protein coding sequences remained unchanged (Fig. 2e). Although increased transcription of LINE elements was observed as a

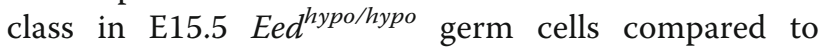
controls, we could not identify specific LINE sequences that were consistently dysregulated. Given the stochastic variation observed in Oct4GFP expression (Fig. 1f, g), and the increased variation between

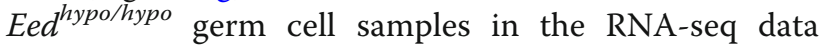
(Additional file 1: Figure S4C), a plausible explanation for this is that a specific LINE element may be affected at one loci in one cell, but not affected in another cell, resulting in variation across the whole cell population.

Despite this caveat, these combined RNA-seq and ChIP-seq data demonstrate that H3K27me3 was substantially redistributed on LINE, SINE and LTR elements and on intergenic and intronic regions, but not on protein-coding genes in germ cells of Eed ${ }^{\text {hypo/hypo }}$ mice. Moreover, although only a subset of retrotransposed LINE elements showed reduced H3K27me3, this class of repeats showed almost 10-fold increased global expres-

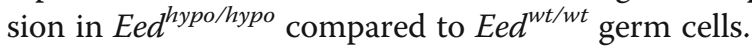

\section{Paternal PRC2 regulates retrotransposed pseudogene silencing in offspring}

The altered H3K27me3 enrichment, increased transcription of retrotransposed elements in foetal male germ cells and the stochastic silencing of the Oct4GFP transgene was strongly suggestive of epigenetic dysregulation in the developing male germ cells. We therefore established a model to investigate whether PRC2-mediated epigenetic dysregulation in the paternal germline might lead to inherited defects in offspring. We hypothesised that sperm developing from diploid germ cells with reduced PRC2 function $\left(E e^{\text {hypo/hypo }}\right)$ would have disrupted epigenetic patterning and produce offspring with altered gene expression profiles. In this model, Eed ${ }^{\text {hypo }}$ hypo males produce Eed $d^{\text {hypo }}$ sperm that develop in the absence of normal EED, while Eed ${ }^{\text {hypo/wt }}$ males produce Eed ${ }^{\text {hypo }}$ sperm that develop in the presence of a normal functioning Eed allele. Based on this differential EED content, mating of these males with normal wild-type females would allow the detection of paternally transmitted epigenetic effects in the absence of any confounding maternal contributions. Critically, comparison of offspring with the same Eed ${ }^{\text {hypo/wt }}$ genotype produced from Eed $d^{\text {hypo/hypo }}$ and Eed ${ }^{\text {hypo/wt }}$ fathers would reveal differences in gene expression due to altered epigenetic patterning in the sperm (Fig. 3). Similarly, epigenetic differences could also exist in sperm produced by Eed ${ }^{w t /}$ wt and $E e d^{h y p o / w t}$ males due to reduced EED function in the germline of Eed ${ }^{\text {hypo/wt }}$ males.

To test this model, independent Eed $d^{\text {hypo/hypo }}, E e d^{\text {hypo/wt }}$ and $E e d^{w t / w t}$ males were mated with $E e d^{w t / w t}$ females of the same background that had never been exposed to

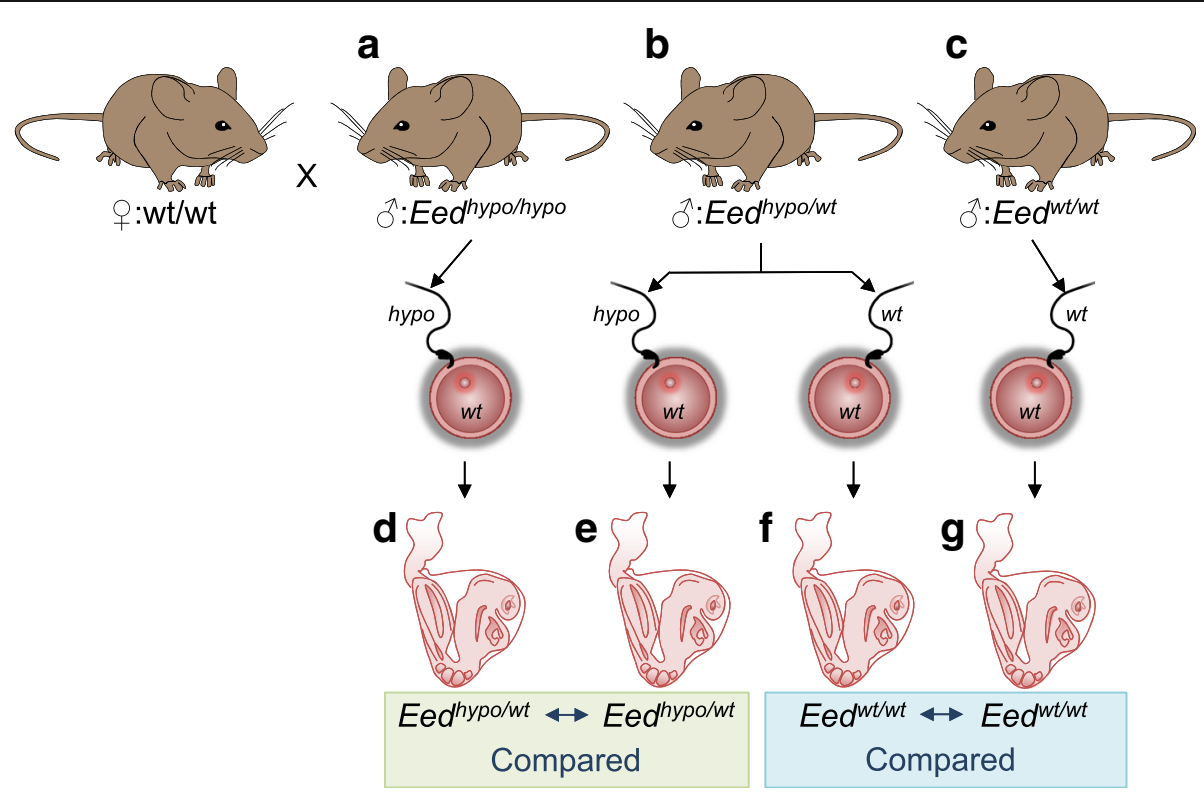

Fig. 3 Breeding and experimental plan to assess epigenetic inheritance. Wild-type (wt/wt) females that had never been exposed to the Eed hypomorphic mutation were mated to either a homozygous (Eed ${ }^{\text {hypo/hypo }}$ ), b heterozygous (Eed hypo/wt) males ( $n=3$ littermate pairs for each genotype) or $\mathbf{c}$ wild-type $\left(\right.$ Eed $\left.^{\text {wtwt }}, n=2\right)$ males. Sperm in heterozygous and wild-type males develop with at least one fully functional copy of Eed (b and $\mathbf{c}$ ), while sperm in homozygous hypomorphic males (a) develop without normally functioning Eed and are expected to contain altered epigenetic patterning. All oocytes are wild-type. $\mathbf{d}$-g Stage- and size-matched E8.5-day embryos were collected for transcriptional analysis. Comparisons between heterozygous embryos ( $\mathbf{d}$ and $\mathbf{e}$ ) and between wild-type embryos ( $\mathbf{f}$ and $\mathbf{g}$ ) identified genes that were misregulated due to non-genetic differences inherited from the sperm 
the Eed mutation (Fig. 3). Eed hypo/hypo $^{\text {and Eed }}{ }^{\text {hypo/wt }}$ brothers produced from three independent mating pairs were used to sire Eed ${ }^{h y p o / w t}$ embryos. Precisely staged and size matched E8.5 heterozygous and wild-type progeny were collected from each cross and photographed. Whole genome gene expression profiles of heterozygous progeny from Eed ${ }^{\text {hypo/hypo }}$ and Eed ${ }^{\text {hypo/wt }}$ males were determined using RNA-seq at a depth of $\sim 40$ million reads per sample $(n=4$ offspring from each group; $40.75 \pm 7$ and $51.3 \pm 13$ million reads per sample for Eed $d^{\text {hypo/hypo }}$ or three $E e d^{h y p o / w t}$ sires, respectively; Fig. 4a).

Comparison of gene expression patterns in E8.5 Eed dypo/ $^{\text {hypol }}$ ${ }^{w t}$ embryos sired by three Eed ${ }^{\text {hypo/hypo }}$ or three Eed $d^{\text {hypo/wt }}$ sibling males (Fig. 4a) identified 1986 differentially expressed transcripts, representing at least 1851 genetically distinct genes separated by more than 5000 bp $(P<0.01$, Benjamini-Hochberg false detection correction) (Fig. 4b).

Of these genes, 779 exhibited greater than 1.5-fold change in expression between Eed $d^{h y p o / w t}$ heterozygous offspring produced by Eed $d^{\text {hypo/hypo }}$ males and Eed $d^{h y p o / w t}$ males. These data demonstrated that there were transcriptional differences between $E e d^{h y p o / w t}$ offspring that result from altered PRC2 function in the paternal germline.

To confirm these changes, Agilent $8 \times 60 \mathrm{~K}$ arrays were used to perform a technically independent assessment of gene expression in the same E8.5 embryos. A total of 234 differentially expressed transcripts, representing 128 distinct genes, were identified between the offspring from Eed ${ }^{\text {hypo/hypo }}$ and $E e d^{\text {hypo/wt }}$ fathers $(P<0.01$; $\geq$ 2-fold change; Benjamini-Hochberg false detection correction) (Fig. 4c). Of these 128 genes, 112 were also identified as differentially regulated in the RNA-seq analysis (Additional file 9: Table S8). Moreover, the direction of change (up- or downregulation) for the transcripts identified by array correlated with the RNA-seq analyses $\left(R^{2}=0.87\right)$ (Additional file 1: Figure S5A). Mapping analyses revealed localization of these genes across all autosomes and the $\mathrm{X}$ chromosome (Additional file 1: Figure S5B). By contrast, comparison of Eed ${ }^{w t / w t}$ embryos produced by $E e d^{h y p o / w t}$ and $E e d^{w t / w t}$ fathers ( $n=4$ each) using Agilent $8 \times 60 \mathrm{~K}$ arrays did not identify any significant differentially expressed genes (Additional file 1: Figure S5C; cut-off: $>2$-fold change and $P<0.01$ with Benjamini-Hochberg false detection correction). This indicates that having at least one wild-type Eed allele is sufficient to support normal paternal epigenetic inheritance.

Gene ontology analysis of the 112 differentially expressed genes identified by the array and RNAseq analyses revealed significant enrichment for processed retrotransposed pseudogenes $\left(P<4 \times 10^{-7}\right.$, Fisher Exact test $)$ and lincRNAs $(P<0.05$, Fisher Exact test) (Fig. $4 \mathrm{~d})$. Further examination using Retrofinder in UCSC (Retroposed Genes V6, UCSC) identified 54 expressed retrotransposed sequences (pseudogenes and pseudogenes/ lincRNA; Fig. 4d). Typically, multiple independent copies of the same parent gene were identified, indicating that these pseudogenes are commonly regulated. In addition, GeneSpring analysis classified 40 probes as
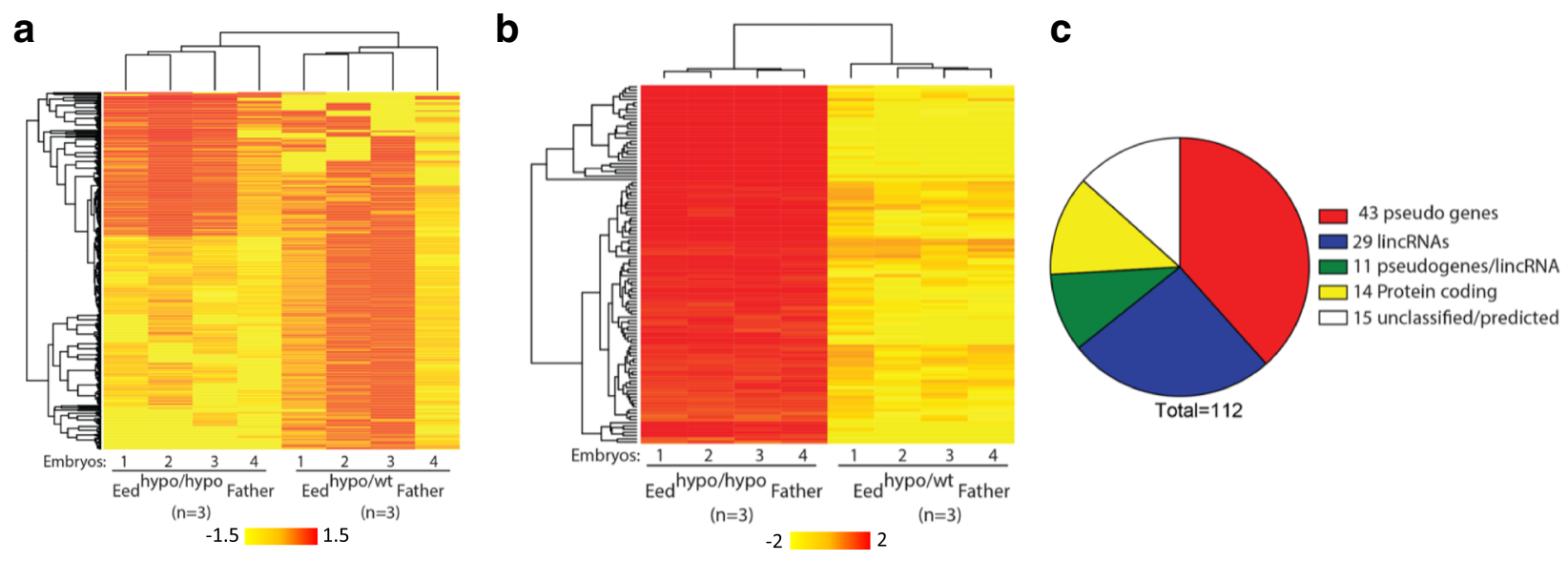

Fig. 4 Global transcription is altered in offspring of Eed hypo/hypo males. a Heatmap of 1986 differentially expressed transcripts $(P<0.01$, $\geq 1.5$-fold change; Benjamini-Hochberg false detection correction) detected using RNA-seq. Each column represents an RNA sample from an individual heterozygous embryo

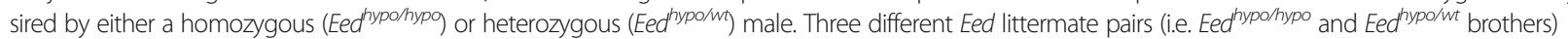
were used to generate progeny for each genotype. b Heat map of 112 differently expressed genes detected using both Agilent 8x60K arrays and RNA-seq $(P<0.01$; $\geq 2$-fold change; Benjamini-Hochberg false detection correction for arrays and RNAseq). Each column (1-4) represents an RNA sample from an individual heterozygous embryo sired by either an Eed ${ }^{\text {hypo/hypo }}$ or an Eed ${ }^{\text {hypo/wt }}$ male. c Pie chart illustrating functional classification of differentially regulated genes detected using micro-array and RNA-seq. Processed retrotransposed pseudogenes $\left(P<4 \times 10^{-7}\right.$, Fisher exact test) and lincRNAs $\left(P<0.05\right.$, Fisher exact test) were significantly enriched in genes upregulated in Eed ${ }^{\text {hypo/wt }}$ progeny of Eed ${ }^{\text {hypo/hypo }}$ compared to Eed ${ }^{\text {hypo/wt }}$ males 
lincRNAs, 11 of which were also classified as retrotransposed pseudogenes, consistent with the established ability of pseudogenes to produce noncoding RNAs [46]. All of these retrotransposed genes and lincRNAs were upregulated in progeny of Eed ${ }^{\text {hypo/hypo }}$ males compared to progeny of Eed hypo/wt males (Fig. 4c), suggesting a primary role for paternal EED in silencing these sequences in the offspring. With the exception of retrotransposed pseudogenes, no differences were detected in expression of other repetitive sequences, including LINE elements.

\section{Paternal PRC2 alters preimplantation cleavage rates and cell cycle gene expression in offspring}

To further investigate the role of paternal germline EED function in embryonic development, we analysed preimplantation development. Zygotes produced by Eed ${ }^{\text {hypo/hypo }}$ and $E e d^{h y p o / w t}$ males were cultured to blastocyst stage and their development documented using automated time-lapse photography of individual embryos. Embryos were imaged every $5 \mathrm{~min}$ facilitating measurement of cleavage rates and embryo development to blastocyst stage. Heterozygous embryos $(n=24)$ sired by Eed ${ }^{\text {hypo/hypo }}$ males underwent $2-4$ cell cleavage $\sim 3 \mathrm{~h}$ earlier than heterozygous $(n=10, P=0.0054)$ or wild-type embryos sired by Eed ${ }^{\text {hypo/wt }}$ males ( $n=12, P=0.0240$; Fig. $5 \mathrm{a}$ ). Consistent with this, time to develop from two-cell to eight-cell embryos was reduced compared to heterozygous and wild-type embryos produced by Eed ${ }^{\text {hypo/wt }}$ males. Time from two-cell to blastocyst was also reduced in heterozygous embryos produced by Eed $d^{\text {hypo/hypo }}$ males but was not significantly different from wild-type embryos produced by Eed ${ }^{\text {hypo/wt }}$ males (Fig. 5a). Collectively, these data show that preimplantation embryos from Eed ${ }^{\text {hypo/hypo }}$ males exhibit impaired development.

Consistent with this, RNA-seq analysis of eight-cell embryos produced by Eed $d^{\text {hypo/hypo }}$ and $E e d^{w t / w t}$ males $(n=5$ pools of $\sim 10$ embryos/sample; $>20$ million reads per sample; Fig. 5b, Additional file 1: Figure S4D-F) revealed 157 transcripts with $>2$-fold increased expression and 109 transcripts with decreased expression in the offspring of Eed hypo/hypo males $(P<0.01$, Benjamini-Hochberg false detection correction) (Fig. 5b; Additional file 10: Table S9 and Additional file 11: Table S10). Examination of these differentially expressed genes using GSEA identified KEGG_Cell_Cycle as the only significantly enriched pathway affected in the preimplantation progeny of Eed ${ }^{h y p o / h y p o}$ and $E e d^{w t / w t}$ males $(q=0.00479)$. Included in this list were five genes, three of which regulate DNA replication and cell cycle progression [Mad2l1 (11.8 fold down), Tdfp2 and Mcm3 (2.2-fold down)] and two that regulate meiotic progression in oocytes [Pkmyt1 (4.7-fold up) and Sme1b (3.8-fold up)] (Fig. 5b, Additional file 10: Table S9 and Additional file 11: Table S10). In addition, six of the top 12 most highly upregulated genes in eight-cell embryos produced by Eed ${ }^{\text {hypo/hypo }}$ males were retrotransposed pseudogenes. However, analysis using HOMER revealed no differences in the expression of LINE elements or other repetitive sequences.

To determine the impacts of depleting paternal EED on peri-natal development, post-natal day (PND) 5 offspring of Eed ${ }^{h y p o / h y p o}$ and $E e d^{h y p o / w t}$ males were weighed, measured and fixed for histopathological analyses. PND5 pups

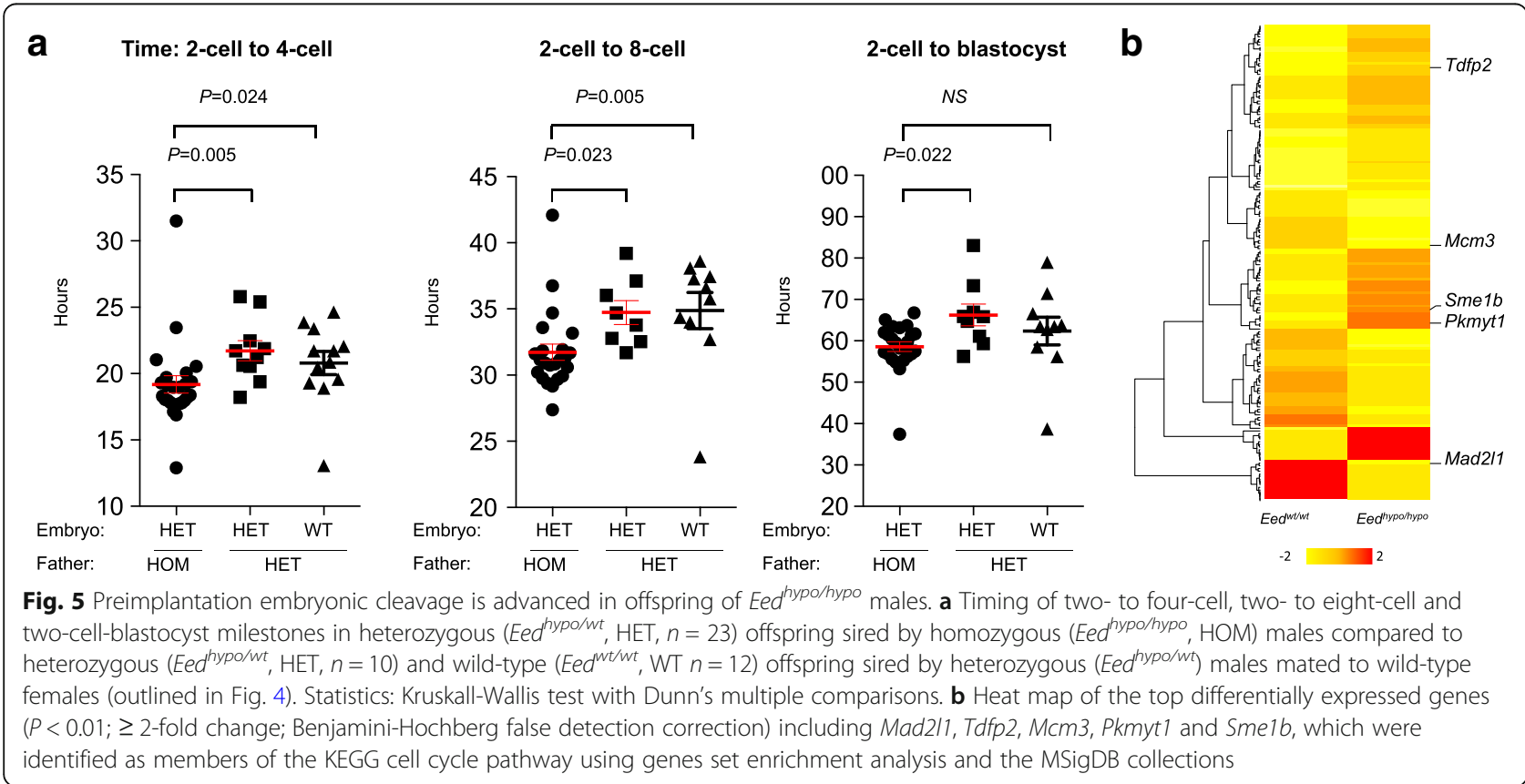


produced by $E e d^{h y p o / h y p o}$ and $E e d^{h y p o / w t}$ males $(n=2$, for each genotype, each crossed with three wild-type females) were not significantly different in weight, crown-rump or nose-rump lengths (6-7 litters for each genotype, $n=56$ and 48, respectively; litter sizes 7-9 pups, with one litter of 6 and 1 litter of 10) (Additional file 1: Figure S6). In addition, histopathological analyses examining 41 tissues were performed on PND5 male heterozygous offspring sired by three sets of sibling Eed ${ }^{\text {hypo/hypo }}$ ( $n=7$ offspring) and $E e d^{h y p o / w t}$ males ( $n=6$ offspring) and identified no obvious phenotypic differences.

Together, these data demonstrated that males lacking EED in the paternal germline produce offspring with altered transcriptional control of retrotransposed pseudogenes and lincRNAs. Moreover, preimplantation development was altered in these offspring, with more rapid cleavage and dysregulated control of cell cycle. Since retrotransposed sequences were also epigenetically dysregulated and over-expressed in the paternal germline, it is likely that reduced function of PRC2 in the paternal germline explains the developmental differences inherited in the offspring of Eed ${ }^{\text {hypo/hypo }}$ males.

\section{Discussion}

With the exception of DNA methylation, establishment of epigenetic information in the germline and its inheritance in the following generation is poorly understood. Recent studies in mice and humans have demonstrated differential enrichment of H3K27me3 at retained nucleosomes in sperm, raising the possibility that PRC2 establishes heritable epigenetic information that significantly affects paternal offspring [34-36]. Here we identify epigenetic and transcriptional changes in the paternal germline of Eed ${ }^{\text {hypo/hypo }}$ males during a key period of paternal epigenetic programming. Moreover, offspring produced by Eed ${ }^{\text {hypo/hypo }}$ males were significantly different from offspring produced by Eed ${ }^{\text {hypo/wt }}$ controls at developmental and molecular levels. Since these offspring were all heterozygous for the Eed mutation, but were derived from sperm that developed with or without normal EED function, these observations provide prima facie evidence that PRC2 mediates epigenetic effects in the paternal germline that alter transcriptional and developmental outcomes in offspring. Consistent with roles for PRC2 in regulating intergenerational inheritance in Drosophila, C. elegans and Xenopus [47-51], our data support a role for PRC2 in regulating epigenetic inheritance in mammals. Moreover, a previous study demonstrated that altered function of the H3K4me3 demethylase in sperm can mediate paternally transmitted transgenerational epigenetic inheritance in mice [6]. Together, these studies strongly indicate that epigenetic inheritance is influenced by histone-modifying enzymes in mammals.
Surprisingly, although survival and male fertility were compromised in Eed hypomorphic animals, low EED function did not significantly alter expression patterns of protein-coding genes in developing male foetal germ cells. Consistent with this, H3K27me3 was not depleted on protein-coding genes in Eed ${ }^{\text {hypo/hypo }}$ male foetal germ cells, perhaps explaining why developmental gene expression remained unaltered. Similarly, in a related study we reduced global H3K27me3 levels by $80 \%$ in male foetal germ cells using the anti-EZH2 drug, GSK126, to treat gonads cultured from E12.5 to E15.5, but no significant changes in transcription of coding genes were detected using expression arrays [40]. Combined, these studies indicate that despite enrichment of H3K27me3 on many developmental genes that are not expressed in germ cells [28], these genes appear to resist upregulation when EED or EZH2 function is compromised and/or H3K27me3 levels are reduced.

Although H3K27me3 enrichment and transcription of coding genes was unaffected in foetal germ cells of Eedhypo/hypo males, H3K27me3 was reduced on a substantial number of LINE, SINE and LTR elements. Moreover, transcription of LINE elements was significantly increased as a class in Eed ${ }^{\text {hypo/hypo }}$ germ cells, although it was not possible to identify individual TE sequences that were transcriptionally altered suggesting that variation in expression may occur at different LINE element loci on a cell to cell basis. Consistent with this, we observed silencing of the Oct4GFP transgene in occasional patches of germ cells in $60 \%$ of male Eed hypo/hypo foetuses, indicating that activity of this transgene is subject to EED-sensitive stochastic cell to cell variation in male germ cells. Combining these observations, we propose that loss in H3K27me3 enrichment across LINE elements in the male germ cells in Eed ${ }^{\text {hypo/hypo }}$ mice leads to derepression of LINE elements, but this occurs in a stochastic pattern in individual cells. The impact of this across the cell population was manifest in significantly increased expression of LINE elements as a class across the cell population. Similar stochastic variation has previously been demonstrated for epigenetic regulatory mechanisms [52] and may be more pronounced in the Eed hypo/hypo model than in global or tissue-specific complete loss of function (e.g. Eed knock out) models in which H3K27me3 is completely removed.

Together, our data highlight significant H3K27me3 enrichment on LINE, SINE and LTR elements in foetal germ cells during the period when DNA methylation begins to be re-established, supporting a role for PRC2 in germline epigenetic programming. In addition, we identified a cohort of retrotransposed pseudogenes that were derepressed in E8.5 progeny of males that had reduced function of EED in the paternal germline. Although less obvious than in the E8.5 progeny, six of the top 12 
upregulated sequences in eight-cell progeny of Eed $d^{\text {hypo/ }}$ hypo males were also pseudogenes. Combined, these observations provide evidence that PRC2 contributes to H3K27me3-mediated repression of LINE elements during epigenetic reprogramming in the paternal germline.

Transposable elements constitute around $45 \%$ of the genome in mammals. Some of these elements retain potential transpositional activity and must be silenced to prevent their activation and random integration in the genome [53, 54]. LINE elements encompass a group of non-LTR retrotransposons which make up around 20\% of the human genome and are common to many eukaryotes [55-57]. A subset of LINE elements still retains the ability for activity and random mutagenesis; hence, strict epigenetic silencing of these sequences is vital for genome integrity [58]. Retrotransposed pseudogenes and retrotransposable elements are created by reverse transcription of processed or unprocessed mRNAs, followed by integration of these sequences back into the genome. These copies are typically imperfect in that they differ from the parent gene and accumulate mutations over time $[46,54,59]$. Our data indicate that PRC2/ H3K27me3 makes an important contribution to silencing these classes of retrotransposable sequences, both in the developing germline and in the paternal progeny. While repressing retrotransposed elements is essential to prevent their mobilisation and random integration into the genome, the requirement for repressing processed pseudogenes is perhaps less obvious as they typically lack their own promoter and the ability to independently retrotranspose. However, transcribed pseudogenes can produce biologically active noncoding RNAs or proteins that have the capacity to alter cell development and function in the host organism $[46,59]$. Silencing or correct transcriptional regulation of retroduplicated sequences is therefore likely to be important to preserve genome function and correct biological processes.

Several lines of evidence indicate that histone modifications are important for regulating repetitive sequence in the paternal germline. Nucleosomes are retained in repetitive sequences in sperm [37], H3K9me3 and H3K27me3 mark LTRs and LINE elements in the foetal germline [26, 29, 30], and the H3K9me3 methylase, SETDB1, is required for repression of a number retroviral elements, including some, but not all, LINE elements [26]. SETDB1 is also required to regulate inherited effects, apparently mediated through DNA methylation [60]. Moreover, LINE elements play a role in pseudogene retrotransposition [61]. Together, these observations indicate a functional link between H3K27me3 in the paternal germline and deregulation of retrotransposed pseudogenes in the offspring of PRC2 mutant males, although the mechanism through which this operates remains obscure. However, we cannot exclude the possibility that the effects mediated through PRC2 and H3K27me3 are indirect, involving other mechanisms such as altered DNA methylation or inheritance of RNAs that mediate effects in offspring.

Eed ${ }^{\text {hypo/hypo }}$ males produced heterozygous offspring that progressed through the two- to four-cell cleavage stage significantly earlier than heterozygous controls or wild-type offspring sired by Eed ${ }^{\text {hypo/wt }}$ males. Consistent with this, cell cycle genes were dysregulated in eight-cell offspring of Eed $d^{\text {hypo/hypo }}$ males. Most notably, Mad2l1, which inhibits cell cycle progression, was decreased 11-fold and in heterozygous progeny of Eed hypo/hypo males compared to progeny of wild-type males, indicating that this gene may regulate the advanced cleavage rate in Eed hypo/hypo progeny. However, the roles of Mad2l1 and other cell cycle genes identified here have not been established in preimplantation embryo cleavage and further work is required to ascertain their functional roles in this process.

Interestingly, germline de novo mutations in either $E E D$ or $E Z H 2$ result in Weaver syndrome, characterised by growth and congenital defects and cognitive deficit in affected humans [62-65]. The maternal/paternal inheritance pattern in Weaver syndrome is poorly understood, although there is some evidence that mutations occur in either the maternal or paternal allele in the germline suggesting that disruption of PRC2 function in either sperm to oocytes may contribute to Weaver syndrome. In this study, partial loss of EED function in the paternal germline was sufficient to mediate significant, though relatively subtle changes in epigenetic and transcriptional regulation in paternal offspring, but not the spectrum of phenotypic characteristics observed in Weaver syndrome patients. Whether greater loss of PRC2 function in male germ cells or in the maternal germline will lead to increased Weaver-like phenotypic changes in mice is yet to be determined. However, loss of EZH2 function in oocytes led to decreased birth weight in mice, rather than increased birth weight typically observed in Weaver syndrome [66].

Surprisingly, despite significant dysregulation of H3K27me3 enrichment on TEs and decreased male fertility, we observed a substantial number of TEs at which H3K27me3 was increased in Eed hypomorphic male germ cells. The reason for this is not known, although one possibility is that that silencing of retrotransposable elements is given functional priority in the germline, even when PRC2 activity is compromised. Such a mechanism may reduce the potential detrimental effects of these elements in the germline and the next generation. This may retain individual fitness for the animal, despite the introduction of epigenetic variation due to altered epigenetic control in the germline, such as that observed in this study. 


\section{Conclusions}

The current study was designed to determine whether EED regulates epigenetic patterning in the paternal germline that subsequently alters outcomes in offspring. This appears to be the case as both regulation of transcription and preimplantation development were altered in offspring of males with reduced EED function. As existing evidence indicates that EED function is restricted to the establishment of H3K27me3 through PRC2, the simplest interpretation of our data is that PRC2 alters epigenetic patterns in sperm that are manifest in offspring. Reduced PRC2 may have altered the establishment of other epigenetic information to compensate for the change in H3K27me3. This could include changes in RNA content in the sperm that could alter gene expression and embryo development $[67,68]$. However, ultimately these changes would occur as a consequence to the original alteration of H3K27me3 due to the reduced function of EED and PRC2. Therefore, this study provides the first functional evidence that the highly conserved histone-modifying complex, PRC2, mediates paternal transmission of inherited effects in mammals. This complements recent evidence that histone modifications play essential roles in regulating inherited disease $[60,69]$, and emphasises the importance of understanding mechanisms that regulate transmission of epigenetic information through the germline inheritance.

\section{Methods}

Mice

Eed hypomorphic (Eedhypo/hypo) mice were generated by inter-crossing c57bl/6:129T2SvJ Eedl7Rn5-1989SB.Oct4GFP

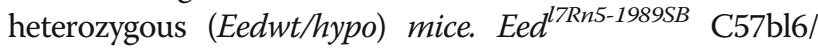
129 mice were maintained under a light-dark cycle in a temperature and humidity-controlled specifically pathogenfree (SPF) facility with access to food and water ad libitum.

\section{Embryo collection and staging}

Animals were time mated and females were inspected for plugs each morning to ensure successful mating. Embryos were collected at fertilisation, 8.5, 12.5 and 15.5 days after the female was plugged.

Zygote to blastocyst development was monitored as previously described [70]. Briefly, embryos collected at fertilisation were kept warm in G-MOPS medium during transfer to the embryo culture facility before washing twice through 50/50 G1/G2 embryo culture media and transferred into $2 \mu \mathrm{l}$ drops of G1/G2 media under oil. Embryos were cultured individually in $6 \% \mathrm{CO}_{2}, 5 \% \mathrm{O}_{2}$ and $89 \% \mathrm{~N}_{2}$ for $96 \mathrm{~h}$ in an incubator (Sanyo MCO 5) equipped with a Primo Vision (Vitrolife, Sweden) Time Lapse Embryo monitoring system allowing morpho-kinetic analysis of embryo development. Morpho-kinetic development of each embryo was documented using time-lapse photography, with images collected every $10 \mathrm{~min}$ for the zygote-blastocyst developmental period. Embryo morphology and cleavage times between zygote to two-cell, two-cell to four-cell, two-cell to eight-cell, and two-cell embryos to blastocyst were documented within Primo Vision and statistically analysed using GraphPad Prism. After culture, embryos were collected and individually snap frozen for genotyping.

To identify differences in transcriptional control in off-

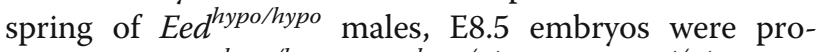
duced by Eed ${ }^{\text {hypo/hypo }}$ Eed $d^{\text {hypo/wt }}$ and $E e d^{w t / w t}$ males mated to wild-type females. Embryos were dissected at E8.5, the physical appearance of each embryo was documented and each embryo was photographed before snap freezing in liquid nitrogen. Photographs and notes were later compared to accurately match samples of the same developmental time points and facilitate accurate gene expression analysis and comparison to controls. All E8.5 embryo samples were kept at $-80{ }^{\circ} \mathrm{C}$ until RNA extraction. E12.5 and E15.5 embryos were examined on collection to ensure they were consistent with E12.5 and E15.5 developmental stage and gonads were dissected. Gonads were fixed for immunofluorescent analysis, or were dissociated and prepared for FACS purification of germ cells.

\section{Genotyping}

The Eed ${ }^{1989} \mathrm{~T}>\mathrm{A}$ point mutation was detected in embryos by reverse transcribing RNA using SuperScript ${ }^{\circ}$ III Reverse Transcriptase Kit (Life Technologies \# 18080051). Samples were PCR amplified using Eed-specific primers (forward: 5' - TCACAGGGGGAGATACGGT TATT and reverse: 5' -CTGACAGGAGAAGGTTTGG GTCT) cleaned using ExoSAP-IT (Affymetrix, 78250) and the cDNA subjected to Sanger sequencing at the MHTP Medical Genomics Facility. Resulting sequences were assessed using FinchTV Geospiza software.

\section{Fertility testing}

A controlled breeding experiment was performed to determine the fertility of the male Eed hypomorphic

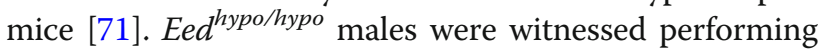
mounting behaviours and successfully produced plugs. The number of pups produced from each female after 1 month was recorded. Each male was housed with two virgin 6-week-old wild-type female mice for 1 month, before replacing the females with another two virgin 6-week-old wild-type females. The number of pups from each female was counted and recorded. If the female was not pregnant after a month, her litter size was counted as 0 . Average litter size from four females was calculated for each male and grouped by genotype. Average group litter size was analysed using 
Bartlett's F-test to compare variances for the three groups and a non-parametric Mann-Whitney test to determine statistical significance. A chi-square test was used to statistically assess differences in the occurrence of successful pregnancies between males grouped by geno-

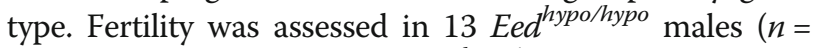
13) along with age-matched Eed ${ }^{h y p o / w t}(n=13)$ and Eed ${ }^{w t /}$ wt $(n=10)$ brothers.

\section{Histology}

Testes from Eed $d^{\text {hypo/hypo }}(n=21)$, Eed $^{\text {hypo/wt }}(n=23)$ and $\operatorname{Eed}^{w t / w t}(n=19)$ males were processed for histology. Each testis was weighed and the testis capsule nicked, immersed in Bouin's fixative overnight, washed three times in $70 \%$ ethanol ( $\mathrm{vol} / \mathrm{vol})$, processed into paraffin wax and stained with periodic acid Schiff (PAS) reagent and haematoxylin. Assessment of testis histology was carried out to determine the presence, or absence, of all germ types and their morphological integrity in comparison with wild-type mice of the same age. Initially, one testis was snap frozen and stored at - 80 for sperm count and hormone assessment while the other testis was used for histological analysis. However, after the observation that there was no difference in DSP, all future gonads were fixed for histology assessment.

\section{Daily sperm production}

Frozen testes from Eed $d^{\text {hypo/hypo }}(n=5)$, Eed ${ }^{\text {hypo/wt }}(n=6)$ and $E e d^{w t / w t}(n=3)$ were allowed to thaw at RT, weighed before a fragment was removed, weighed, decapsulated and homogenised in $600 \mu \mathrm{l}$ of SMT solution. Ten microlitres of homogenate was placed on each side of the haemocytometer. The average number of sperm heads was calculated from counting 80 small squares on both sides of the haemocytometer. Daily sperm counts were calculated as previously reported [72, 73]. Briefly, the volume of homogenate, weight of the sample fragment and total weight of the testis were used to calculate the total number of spermatids per testis. As developing spermatids spend 4.84 days in steps 14-16 during spermatogenesis, the values for the number of spermatids per testis were divided by 4.84 to obtain daily sperm production. Statistical significance was determined using one-way ANOVA with Tukey's multiple comparison, with $P<0.05$ considered significant.

\section{Immunofluorescence}

Embryos were harvested at E12.5 and E14.5, sexed based on gonad morphology or via PCR [74]. Foetal gonads were isolated and fixed at RT in PBS containing 4\% paraformaldehyde for 20 or 75 min respectively. Gonads were washed three times in PBS and cryoprotected in $30 \%$ sucrose in PBS overnight and mounted in optimal cutting temperature (OCT). Cryosections were cut at $8 \mu \mathrm{m}$, permeabilised with $1 \%$ Triton-X and non-specific staining blocked with $5 \%$ BSA. Immunofluorescence staining was carried out as described [75, 76]. EED (R\&D Technologies, AF5827, diluted 1/100), EZH2 (Cell Signalling Technology D2C9, diluted 1/400), SUZ12 (Cell Signalling Technology D39F6, diluted 1/100), H3K27me3 (Cell Signalling Technologies, C36B11, diluted 1/400), OCT4 (Santa Cruz sc8628, diluted 1/500), DPPA4 (R\&D Systems AF3730, diluted 1/400) and MVH/DDX4 (Cell Signalling Technology \#8761, diluted $1 / 300)$ primary antibodies were each diluted in PBS containing $1 \%$ BSA incubated for $1 \mathrm{~h}$ at RT. Donkey anti-goat, sheep or rabbit Alexa-594 (Life Technologies) secondary antibodies were used at 1/500 dilution, while eGFP fluorescence was detected directly in the 488-nm channel. To assess non-specific staining, additional sections were analysed using secondary antibody only controls. Images were obtained using a Nikon ${ }^{\circ} \mathrm{C} 1$ confocal microscope. Images were visually analysed using ImageJ (version: 2.0.0-rc-19/1.49m). All IF experiments were replicated using three to five pairs of gonads per genotype.

\section{Flow cytometry}

Pregnant mothers were injected intraperitoneally (i.p.) with $20 \mathrm{mg} / \mathrm{kg}$ 5-ethynyl-2-deoxyuridine (EdU) to facilitate in vivo analysis of gonadal cell proliferation. Flow cytometry was performed as previously described [40, 77, 78]. Dissociated gonadal cells were stained using antibodies specific for SOX9 and AMH allowing identification of Sertoli cells and SOX9 (Millipore AB5535, diluted 1/300) and AMH (Santa Cruz sc-6886, diluted $1 / 200$ ) staining intensity in the Sertoli cell population. Cell cycle was measured in $E e d^{w t / w t}, E e d^{w t / h y p o}$ and Eed ${ }^{\text {hypo/hypo }}$ samples as previously described [77, 78]. Germ cells were identified using an antibody specific for MVH (R\&D Systems, AF2030 diluted 1/100; [77, 78]).

\section{Fluorescence-activated cell sorting}

Fluorescence activated cell sorting was performed essentially as described [42, 75, 76]. Foetal gonads were collected at E15.5 and sexed based on gonadal morphology. Male gonad pairs were dissociated to single cells in $0.25 \%$ trypsin containing EDTA. Trypsin activity was blocked by adding $500 \mu \mathrm{l}$ DMEM containing 10\% FBS. The cells were filtered through an $80-\mu \mathrm{m}$ nylon mesh (BD Biosciences), pelleted and resuspended in $300 \mu \mathrm{l}$ PBS containing $0.4 \%$ BSA for FACS. GFP-positive (germ cells) and GFP-negative (somatic cells) were collected at $>95 \%$ purity using a BD Influx Cell Sorter (BD Biosciences). Propidium iodide (200 ng/ml) was added to cell suspensions to monitor cell viability, and only viable 
cells (>95\%) were collected. Germ cells were pelleted and either fixed for ChIP or snap frozen for RNA. Each gonad pair yielded approximately 20,000 germ cells, and similar proportions of GFP-positive germ cells were isolated from Eed ${ }^{w t / w t}, E e d^{w t / h y p o}$ and Eed $d^{\text {hypo/hypo }}$ foetuses (Fig. 1h). We were unable to isolate any Oct4GFP-negative germ cells.

\section{RNA extraction and quality assessment}

RNA was extracted from E8.5 embryos using the Genelute Mammalian total RNA miniprep kit (Sigma, RTN70-1KT), DNAse treated using TURBO DNA-free ${ }^{\mathrm{TM}}$ Kit (Ambion, AM1907) and purified from the DNAse reaction using Agencourt RNAClean XP (Beckman coulter, A63987). RNA was extracted from pools of eight to ten carefully staged eight-cell embryos, with each pool representing separate litters using Agencourt RNAClean XP (Beckman coulter, A63987) chemistry and the RNA DNAase treated on the beads, before elution, freezing and storage at $80{ }^{\circ} \mathrm{C}$. Isolation of E15.5 germ cells was performed by FACS purifying approximately 15,000 germ cells from each pair of E15.5 foetal testes. RNA was extracted from the germ cells using the RNeasy Micro Kit (Qiagen) including an on column DNAse step. For all samples isolated from E8.5 embryos, E15.5 germ cells and eight-cell embryos, RNA quantity and quality were measured using a Qubit $^{\circledR}$ RNA HS Assay Kit and the Qubit ${ }^{\oplus}$ 2.0 Fluorometer (Life Technologies, Q32866) and an Agilent 2100 Bioanalyzer (Agilent Technologies, Santa Clara, CA). Only samples with a RIN score above 8 were used for RNA sequencing and microarray analysis.

\section{Quantitative real-time RT-PCR (qRT-PCR) using Fluidigm biomark}

RNA was extracted form 50-200K FACS purified E15.5 testis somatic cells or from whole E12.5 gonads using $600 \mathrm{ml}$ of Trizol in $2 \mathrm{Ml}$ heavy phase-lock tubes. DNAsed using Ambion Turbo kit and cDNA synthesised using super script III (Invitrogen) kit. Gene expression was analysed using real-time quantitative polymerase chain reaction with BioMark HD technology (Fluidigm) 96.96 Dynamic Array IFCs (Fluidigm). The geometric CT mean of reference genes Canx, Sdha, and Mapk1 was used to calculate the relative gene expression using the delta-delta CT method. We have shown previously that Canx, Sdha, and Mapk1 are expressed at stable levels in E12.5-E15.5 foetal germ cells and somatic cells [75]. Statistical significance was determined using one-way ANOVA with Tukey's multiple comparison test for $E e d^{\text {hypo/hypo }}(\mathrm{E} 12.5 n=7$, E15.5 $n=14), E e d^{\text {hypo } / w t}$ (E12.5 $n=7, \mathrm{E} 15.5 n=12)$ and $\operatorname{Eed}^{w y / w t}(\mathrm{E} 12.5 n=5$, E15.5 $n=11)$. Where variances were unequal, a non-parametric test was used (Mann-Whitney). A $P$ value of less than 0.05 was considered significant.

\section{RNA sequencing}

RNA sequencing libraries were constructed from $50 \mathrm{ng}$, $25 \mathrm{ng}$ or $\sim 2 \mathrm{ng}$ of total RNA isolated from E8.5 embryos, E15.5 germ cells or eight-cell embryos, respectively. RNA sequencing libraries were constructed using Nugen Mondrian SPIA Library preparation using Nugen protocol M01335v2 after ribosomal depletion of total RNA using the RiboZero kit (Illumina, RS-122-2201). RNA sequencing was performed on an Illumina HiSeq 1500 instrument with condition sequenced in four biological replicates, derived from $\geq 3$ independent sires (for E8.5 and eight-cell embryos) or from four embryos from multiple litters (E15.5 germ cells). Quality filtering and adaptor removal was performed using the Trimmomatic software tool with default trimming parameters [79]. Reads were mapped to the mouse genome, version mm10, using TopHat [80] and genes identified and compared to Ensembl using the RNA-eXpress analysis tool [81]. Replicate quality was assessed by applying multiple dimensional scaling plots, and differential expression analysis was performed using the voom-limma analysis workflow applying empirical Bayes F-test [82]. Transcript enrichment was performed based on HOMER-assembled annotations with statistical significance of enrichment assessed by Fisher exact test with Benjamini-Hochberg false detection correction. Pseudogene classification was performed using RetroFinder program (Retroposed Genes V6, UCSC). Data was visualised using the ggplot package within the $R$ analysis environment. Gene set enrichment analysis was performed using MSigDB collections [83] with enrichment assessed by Fisher exact test with Benjamini-Hochberg. Sequence data is available through the European Nucleotide Archive (ENA) accession numbers ERP106776 (E15.5 germ cell data), ERP010195 (8.5-day embryo data) and ERP013725 (eight-cell embryo data).

\section{Expression microarray analysis}

RNA labelling was performed using the one-colour Low RNA Input Quick Amp Amplification Kit (Agilent) and hybridised to the SurePrint G3 Mouse GE 8x60K microarrays according to the manufacturer's instructions (Agilent). Slides were scanned on an Agilent High-Resolution C DNA Microarray Scanner (G2505B), the features extracted with Feature Extraction 11.0.1.1 and the data analysed to obtain background-subtracted, spatially detrended processed signal intensities. Agilent flagged features were excluded from further analysis. Differential expression was assessed in GeneSpring GX version 12 (Agilent). Percentile shift normalisation was performed, and the probe sets were 
filtered to exclude the lowest $20 \%$ of probes and those not expressed in at least four of the eight (Eed ${ }^{w t / h y p o}$ vs $E e d^{w t /}$ hypo offspring-array 1) or eight $\left(E e d^{w t / w t}\right.$ vs $E e d^{w t / w t}$ offspring-array 2) samples. Differential expression was reported where twofold change was observed applying a 0.01 FDR after Benjamini-Hochberg multiple testing correction. Data was visualised in a hierarchically clustered heat map within the GeneSpring software package. Raw data is available through the NCBI Gene Expression Omnibus GSE68213 (composed of GSE68212 and GSE68211).

\section{Chromatin immunoprecipitation (ChIP)}

ChIP was performed on approximately 20,000 FACS purified germ cells from individual $\operatorname{Eed}^{w t / w t}(n=4)$ and Eedhypo/hypo $(n=4)$ embryos using low input ChIP $[84,85]$ with minor alterations. Briefly, cells were cross-linked in $1 \%$ formaldehyde/PBS for $5 \mathrm{~min}$ before adding glycine/ PBS at a final concentration of $125 \mathrm{mM}$. Cells were pelleted and washed in PBS and stored at $-80{ }^{\circ} \mathrm{C}$. Dynabeads were washed in RIPA buffer and bound to $2.4 \mu \mathrm{g}$ of H3K27me3 rabbit polyclonal (Millipore cat\# 07-449, $1 \mathrm{mg} / \mathrm{ml}$ ) or IgG rabbit CST (cat\#3900S, $2.5 \mathrm{mg} / \mathrm{ml}$ ) by incubating for a minimum of $2 \mathrm{~h}$ at $4{ }^{\circ} \mathrm{C}$ at $40 \mathrm{rpm}$.

Cells were thawed on ice and lysed in $13 \mu \mathrm{l}$ of lysis buffer for $3 \mathrm{~min}$, vortexed for two 5-s bursts at room temperature and $117 \mu \mathrm{l}$ modified RIPA buffer containing Tris-HCl pH 7.5 EGTA, Triton X-100 $0.1 \%$ SDS. Sonication conditions were pre-optimised by assessing germ cell-derived sonicated chromatin samples on a gel and via bioanalyser High Sensitivity DNA Analysis Kits (Agilent Technologies) to ensure that the majority of chromatin fragments yielded were within a 300-100-bp range. Germ cell chromatin was sonicated for $10 \mathrm{~min}$ at peak power 105 , duty factor 2.0 , cycles/burst 200 using a Covaris s220 instrument. Sample was recovered and $5 \mathrm{M}$ $\mathrm{NaCl}$ added to a final concentration of $140 \mathrm{mM}$ (equivalent to RIPA buffer). A $10 \mu \mathrm{l}$ aliquot of each sample was removed for an input control and the remaining chromatin solution immediately transferred to the antibody-bead complexes. The chromatin was incubated with the antibody-bead suspension at $4{ }^{\circ} \mathrm{C}$ rotating at $40 \mathrm{rpm}$ for $2 \mathrm{~h}$ before washing in cold RIPA buffer and resuspending TE. To reverse crosslink, chromatin-bead suspensions and input controls were resuspended in elution buffer containing freshly added SDS and Proteinase $\mathrm{K}$ and incubated for $2 \mathrm{~h}$ at $68{ }^{\circ} \mathrm{C} 13,000 \mathrm{rpm}$. DNA was purified for sequencing using Agencourt Ampure XP beads (Beckman Coulter).

H3K27me3 rabbit polyclonal (Millipore cat\# 07-449, $1 \mathrm{mg} / \mathrm{ml}$ ) antibody specificity was validated using Active Motif Modified Histone Array analysis (Catalogue number 13001) according to the array manufacturer's protocol, with the exception that dilution of the primary antibody (1/2000) was as recommended by
Millipore for western blotting. Mouse anti Rabbit HRP secondary antibody was used at the recommended dilution (1/2500). Imaging of the array was performed using incremental accumulating exposures to ensure data was collected in the linear detection range. Data analysis was performed using Array Analyse Software (Active Motif) and the specificity factor determined for the antibody with reference to 384 histone peptide variants on the array (Additional file 1: Figure S7).

\section{ChIP sequencing and analysis}

ChIP-Seq Libraries were prepared from using the Ovation Ultralow System V2 using Nugen protocol M01379v1. Each library was quantitated using a Qubit instrument and the DNA size profile determined using an Agilent Bioanalyzer. Libraries were finally quantitated by qPCR, pooled in an equimolar ratio and all eight libraries run on one lane of a HiSeq1500 sequencer to obtain 50-bp single end reads. Raw reads were bioinformatically separated into individual libraries and trimmed using the trimmomatic tool [79] before mapping. Sequences were mapped to the mouse genome (mm10) using bowtie2, resulting in over 95\% quality mappable reads. PCR duplicates were marked in the mapping files (BAM format) for filtering in further steps, ensuring use of unique reads during peak identification. Peak identification and differential peak identification was performed using HOMER (http://homer.salk.edu/homer/).

Differential peaks were annotated using HOMER, allowing all peaks to be assigned to specific sequence classes. Genomic sequence classes outside repeats were analysed for over-, under- or expected representation in sequences differentially enriched for H3K27me3 in Eedhypo/hypo germ cells compared to Eed ${ }^{w t / w t}$ germ cells. Initially, the expected number of peaks was calculated by determining the proportion of the genome covered by each sequence class (e.g. for intergenic sequences $813,929,088 \mathrm{bp}$ ) divided by the total annotated genome size (i.e. $813,929,088 / 2,631,564,759=30.93 \%$ ). The number of peaks in each sequence class (e.g. for intergenic sequences with increased H3K27me3 in Eed hypo $^{\text {hy }}$ hypo germ cells: 380 ) was divided by the total number of peaks for which increased H3K27me3 was detected (e.g. peaks with increased H3K27me3: 1158). Over-, under- or expected representation was determined by calculating whether the actual percentage represented in the differentially enriched sequences (e.g. for intergenic sequences with increased H3K27me3: 380/1158 $=41.17 \%)$ significantly differed from the expected values (for intergenic sequences $=30.93 \%$ ) based on genome-wide representation for each sequence class using a chi-square test to compare expected and observed values. ChIPseq data is available through the 
NCBI Gene Expression Omnibus accession number GSE110529.

Representation of repeat sequences was determined by determining the numbers of repeat sequences annotated in each sequence category compared to the total number of repeats in the genome to provide the expected representation of repeats in each repeat category annotated in UCSC repeat masker. A hypergeometric analysis was performed to determine representation of each repeat category in all H3K27me3 peaks identified in $E e d^{w t / w t}$ and $E e d^{h y p o / h y p o}$ germ cells and in peaks that had altered H3K27me3 in Eed ${ }^{w t / w t}$ compared to Eedhypo/hypo germ cells, and vice versa.

ChIP data from the current study was compared with that reported by $\mathrm{Mu}$ et al. [32] and $\mathrm{Ng}$ et al. [29] in which H3K27me3 peaks were identified using MACS. Polycomb target genes from Boyer et al. [86] were transposed to the current $\mathrm{mm} 10$ nomenclature and H3K27me3 peaks mapped in the genic and upstream regions of these Polycomb target genes in the current study and studies from $\mathrm{Mu}$ et al. [32] and $\mathrm{Ng}$ et al. [29].

\section{Phenotypic analyses of offspring}

Two Eed hypomorphic males from each genotype (Eed $d^{\text {hypo/hypo }}$ and $E e d^{\text {hypo/wt) }}$ were each bred with three $\mathrm{C} 57 \mathrm{Bl} / 6 \mathrm{~J}$ females, to obtain seven and six litters, respectively. Offspring from $\mathrm{Eed}^{\text {hypo/hypo }}(n=56)$ and Eed ${ }^{\text {hypo/wt }}(n=48)$ fathers were weighed at post-natal day 5; crown-rump and nose-rump measurements were obtained. Offspring from $E e d^{h y p o / w t}$ fathers were genotyped via Transnetyx to analyse wild-type and heterozygous groups. All litters analysed consisted of six to ten pups. One-way ANOVA plus Tukey's post hoc multiple comparisons test was used to statistically analyse the data. A comprehensive phenotypic analysis was also performed using the Australian Phenomics Network Histopathology and Organ Pathology Service. Neonates were weighed and crown-rump and nose-rump lengths collected. The neonates were decapitated, injected with Bouin's fixative into the thorax and abdomen as well as immersion fixation for $48 \mathrm{~h}$, washed and stored in 70\% EtOH. Analysis included evaluation of all thoracic and abdominal organs, skeletal tissue, nasal/oral region, brain, eyes and auditory/vestibular apparatus. Reports were generated for each neonate, including detailed histopathological and neuropathology descriptions.

\section{Additional files}

Additional file 1: Figure S1. Survival and fertility of Eed ${ }^{\text {hypo/hypo }}$ animals. Figure S2. Foetal somatic and germ cells reached expected developmental milestones and expressed endogenous germ cell markers in Eed hypo/hypo males. Figure S3. H3K27me3 was enriched at known PRC2 target genes in Eed ${ }^{\text {hypo hhypo }}$ compared to Eed ${ }^{\text {wt }}$ /wt germ cells. Figure S4. Read counts and technical consistency between RNAseq samples generated from (A-B) E15.5 Eed $^{\text {wt Wt }}$ and Eed ${ }^{\text {hypo hypo }}$ male germ cells and (C) D8-cell offspring from Eed ${ }^{\text {wt }}$ wt and Eed ${ }^{\text {hypo/hypo }}$ males. Figure S5. Transcriptional analyses of E8.5 offspring produced by Eed hypo/hypo, Eed ${ }^{\text {hypo/wt }}$ and Eed ${ }^{\text {wt }}$ wt male mice mated to wild-type females. Figure $\mathbf{S 6}$. Neonatal weight and size are not different in offspring of Eed ${ }^{\text {hypo/wt }}$ Eed ${ }^{\text {hypo/hypo }}$ and males. Figure S7. Active Motif Modified Histone Array analysis of H3K27me3 ChIP antibody. (PDF $15192 \mathrm{~kb}$ )

Additional file 2: Table S1. Eed ${ }^{\text {wt } / \text { t }}$ germ cell H3K27me3 ChIP-seq peaks. (XLSX $6770 \mathrm{~kb}$ )

Additional file 3: Table S2. Eed ${ }^{\text {hypo/hypo }}$ germ cell H3K27me3 ChIP-seq peaks. (XLSX $8636 \mathrm{~kb}$ )

Additional file 4: Table S3. Comparison of known PRC target genes in ES cells with H3K27me3 peaks in foetal germ cells, ES cells and spermatocytes. (XLSX $1161 \mathrm{~kb})$

Additional file 5: Table S4. Relative representation of annotated repeat categories in H3K27me3 peaks in Eed ${ }^{\text {wt } / \text { t }}$ and Eed hypo/hypo $_{\text {germ }}$ cells. (XLSX $1124 \mathrm{~kb}$ )

Additional file 6: Table S5. ChIP-seq data from E15.5 male Eed ${ }^{\text {hypo/hypo }}$ compared to Eed ${ }^{\text {wt } / \text { wt }}$ germ cells: Peaks with decreased H3K27me3 in Eedhypo/hypo germ cells. (XLSX $70 \mathrm{~kb}$ )

Additional file 7: Table S6. ChIP-seq data from E15.5 male Eed hypo/hypo compared to Eed ${ }^{\text {wt }}$ wt germ cells: Peaks with increased H3K27me3 in Eedhypo/hypo germ cells. (XLSX $83 \mathrm{~kb}$ )

Additional file 8: Table S7. ChIP-seq data showing regions with decreased H3K27me3 in Eed hypo/hypo compared Eed ${ }^{\text {Nt wht }}$ to germ cells identified using EdgeR. (XLSX $11 \mathrm{~kb}$ )

Additional file 9: Table S8. RNA-seq and expression microarray data showing genes differentially expressed in heterozygous E8.5 day embryos sired by Eed ${ }^{\text {hypo/hypo }}$ fathers compared to E8.5-day embryos sired by Eed ${ }^{\text {hypo/wt }}$ fathers. (XLSX $22 \mathrm{~kb}$ )

Additional file 10: Table S9. RNA-seq data showing genes significantly downregulated in eight-cell embryos sired by Eed hypo/hypo fathers. (XLSX $20 \mathrm{~kb}$ )

Additional file 11: Table S10. RNA-seq data showing genes significantly upregulated in eight-cell embryos sired by Eed ${ }^{\text {hypo/hypo }}$ fathers. (XLSX $23 \mathrm{~kb}$ )

\section{Abbreviations}

AMH: Anti-Mullerian hormone; ChIP-seq: Chromatin immunoprecipitation followed by genome wide sequencing; DPPA4: Developmental Pluripotency Associated gene 4; DSP: Daily sperm count; E: Embryonic day; EED: Embryonic ectoderm development; eGFP: Enhanced Green Fluorescent protein;

EZH1: Enhancer of Zeste 1; EZH2: Enhancer of Zeste 2; FACS: Fluorescenceactivated cell sorting; H3K27me3: Trimethylated lysine 27 on histone 3; lincRNA: Long non-coding RNA; LINE: Long Interspersed Nuclear Element; LTR: Long Terminal Repeat; Mad211: Mitotic Arrest Deficient 2 Like 1; Mcm3: Minichromosome Maintenance Complex Component 3; MVH: Mouse Vasa Homologue (also known as DDX4); Nsd: No significant difference; OCT4: Octamer Binding Transcription Factor 4 (also known as Pou5f1); Pkmyt1: Protein Kinase Membrane Associated Tyrosine/Threonine 1; PND: Post-natal day; PRC2: Polycomb Repressive Complex 2; RNAseq: Genome-wide RNA sequencing; Sdha: Succinate Dehydrogenase Complex, subunit A; SINE: Short Interspersed Nuclear Element; SOX9: SRY Box Containing Gene 9; SUZ12: Suppressor of Zeste 12; TEs: Transposable elements

\section{Acknowledgements}

The authors thank Jodee Gould, Trevor Wilson, Vivien Vasic and Denis Cleven at the MHTP Medical Genomics Facility for their help processing samples and Nicolle Gibson, Laura Deans and other staff of the Monash Medical Centre Animal Facilities (MMCAF). Thank you also to Sanna Barrand for contributions to ChIP-seq development, Roxanne Legaie form the Monash Bioinformatics Platform for initial analysis of ChIP-seq data, Dr. Marnie Blewitt for sharing the Eed ${ }^{17 R n 5-19895 B}$ mouse strain, and Dr. Kirsten Hogg, and other members of the Centre for Genetic Diseases at the Hudson Institute for Medical Research, for helpful comments and discussion. We thank Tina 
Cardamone and the Australian Phenomics Network Histopathology and Organ Pathology Service for mouse phenotyping. The authors also thank Atma Ivancevic, Adelaide University, for access to resources used in the ChIP analysis of LINE elements, and Mai Truong, University of Melbourne, with assistance with time-lapse analysis. The authors acknowledge the facilities and scientific and technical assistance of the Monash Histology Platform, FlowCore and Monash Micro Imaging, Department of Anatomy and Developmental Biology, Monash University.

\section{Funding}

PSW was funded by the National Health and Medical Research Council (NHMRC) project grants 1043939 and 1051223 and funding from the Hudson Institute of Medical Research. MOB was supported funded by NHMRC Fellowship 1058356. SCF was supported by an NHMRC Early Career Fellowship 1091097 and Wellcome Trust funding 098051. This work was also supported by the Victorian Government Operational Infrastructure Support Scheme.

\section{Availability of data and materials}

All genome-wide data are available through the following accession numbers. RNA sequencing data are available through the European Nucleotide Archive: PRJEB24910; ERP 106776-E15.5 Germ cell data, PRJEB9120; ERP010195-E8.5-day embryo data, PRJEB12268; ERP013725-8-cell embryo data. ChIP Sequencing data and microarray data are available through the NCBI Gene Expression Omnibus: GSE110529—E15.5 Germ cell ChIP Seq data, GSE68213 (composed of GSE68212 and GSE68211) -E8.5-day embryo microarray data. Other data generated or analysed during this study are included in this article and its supplementary information files.

\section{Authors' contributions}

PSW conceived and designed the study, obtained financial support, analysed the data and drafted the manuscript. JMS contributed to the design, collected and processed the samples, optimised and performed experiments, analysed the data and drafted the manuscript. SCF analysed the data and drafted the manuscript. ZQ, DA and SW performed analysis of ChIP-seq data. $L P, D G$ and $M O B$ contributed to data collection and manuscript preparation. All authors read and approved the final manuscript.

\section{Ethics approval}

No human samples or subjects were used in this study. All experimental manipulations using animals were performed during the animal's active cycle in accordance with the guidelines and regulations of the Monash University Animal Ethics Committee permit number MMCA 2013/33.

\section{Competing interests}

The authors declare that they have no competing interests.

\section{Publisher's Note}

Springer Nature remains neutral with regard to jurisdictional claims in published maps and institutional affiliations.

\footnotetext{
Author details

${ }^{1}$ Centre for Reproductive Health, Hudson Institute of Medical Research, Clayton, Victoria 3168, Australia. ${ }^{2}$ Department of Anatomy and Developmental Biology, Ovarian Biology Laboratory, Biomedicine Discovery Institute, Monash University, Melbourne 3168, Australia. ${ }^{3}$ Host-Microbiota Interactions Laboratory, Wellcome Trust Sanger Institute, Hinxton CB10 1SA, UK. ${ }^{4}$ Centre for Innate Immunity and Infectious Diseases, Hudson Institute of Medical Research, Clayton, Victoria 3168, Australia. ${ }^{5}$ Molecular and Translational Science, Monash University, Clayton, Victoria 3168, Australia. ${ }^{6}$ Bioinformatics and Computational Genetics, School of Biological Sciences, The University of Adelaide, Adelaide, South Australia 5005, Australia. ${ }^{7}$ Schoo of Biological Sciences, Monash University, Clayton, Victoria 3168, Australia. ${ }^{8}$ School of BioSciences, University of Melbourne, Parkville, Australia. ${ }^{9}$ Department of Human Genetics, Leiden Genome Technology Centre, Leiden University Medical Center, Leiden, the Netherlands.
}

Received: 10 July 2018 Accepted: 28 August 2018 Published online: 20 September 2018

\section{References}

1. Prokopuk L, Western PS, Stringer JM. Transgenerational epigenetic inheritance: adaptation through the germline epigenome? Epigenomics. 2015:7(5):829-46.

2. Youngson NA, Whitelaw E. Transgenerational epigenetic effects. Annu Rev Genomics Hum Genet. 2008:9:233-57.

3. Daxinger $L$, Whitelaw E. Understanding transgenerational epigenetic inheritance via the gametes in mammals. Nat Rev Genet. 2012;13(3):153-62.

4. Grossniklaus U, Kelly B, Ferguson-Smith AC, Pembrey M, Lindquist S. Transgenerational epigenetic inheritance: how important is it? Nat Rev Genet. 2013;14(3):228-35.

5. Stringer J, Barrand S, Western P. Fine-tuning evolution: germ-line epigenetics and inheritance. Reproduction. 2013;146(1):R37-48.

6. Siklenka K, Erkek S, Godmann M, Lambrot R, McGraw S, Lafleur C, Cohen T, Xia J, Suderman M, Hallett M, et al. Disruption of histone methylation in developing sperm impairs offspring health transgenerationally. Science. 2015:350(6261):aab2006.

7. Inoue A, Jiang L, Lu F, Suzuki T, Zhang Y. Maternal H3K27me3 controls DNA methylation-independent imprinting. Nature. 2017:547(7664):419-24.

8. Bracken AP, Dietrich N, Pasini D, Hansen KH, Helin K. Genome-wide mapping of Polycomb target genes unravels their roles in cell fate transitions. Genes Dev. 2006;20(9):1123-36.

9. Pasini D, Bracken AP, Hansen JB, Capillo M, Helin K. The Polycomb group protein Suz12 is required for embryonic stem cell differentiation. Mol Cell Biol. 2007;27(10):3769-79.

10. Pasini D, Bracken AP, Jensen MR, Lazzerini Denchi E, Helin K. Suz12 is essential for mouse development and for EZH2 histone methyltransferase activity. EMBO J. 2004;23(20):4061-71.

11. Faust C, Lawson KA, Schork NJ, Thiel B, Magnuson T. The Polycomb-group gene eed is required for normal morphogenetic movements during gastrulation in the mouse embryo. Development. 1998;125(22):4495-506.

12. O'Carroll D, Erhardt S, Pagani M, Barton SC, Surani MA, Jenuwein T. The Polycomb-group gene Ezh2 is required for early mouse development. Mol Cell Biol. 2001:21(13):4330-6.

13. Wang J, Mager J, Schnedier E, Magnuson T. The mouse PCG gene eed is required for Hox gene repression and extraembryonic development. Mamm Genome. 2002;13(9):493-503.

14. Mu W, Starmer J, Fedoriw AM, Yee D, Magnuson T. Repression of the somaspecific transcriptome by Polycomb-repressive complex 2 promotes male germ cell development. Genes Dev. 2014;28(18):2056-69.

15. Schumacher A, Faust C, Magnuson T. Positional cloning of a global regulator of anterior-posterior patterning in mice. Nature. 1996. 384(6610):648

16. Montgomery ND, Yee D, Chen A, Kalantry S, Chamberlain SJ, Otte AP, Magnuson T. The murine Polycomb group protein Eed is required for global histone H3 lysine-27 methylation. Curr Biol. 2005;15(10):942-7.

17. Rinchik EM, Carpenter DA. N-ethyl-N-nitrosourea-induced prenatally lethal mutations define at least two complementation groups within the embryonic ectoderm development (eed) locus in mouse chromosome 7. Mamm Genome. 1993:4(7):349-53.

18. Seisenberger S, Andrews S, Krueger F, Arand J, Walter J, Santos F, Popp C, Thienpont B, Dean W, Reik W. The dynamics of genome-wide DNA methylation reprogramming in mouse primordial germ cells. Mol Cell. 2012; 48(6):849-62.

19. Hackett JA, Reddington JP, Nestor CE, Dunican DS, Branco MR, Reichmann J, Reik W, Surani MA, Adams IR, Meehan RR. Promoter DNA methylation couples genome-defence mechanisms to epigenetic reprogramming in the mouse germline. Development. 2012;139(19):3623-32.

20. Hackett JA, Sengupta R, Zylicz JJ, Murakami K, Lee C, Down TA, Surani MA Germline DNA demethylation dynamics and imprint erasure through 5hydroxymethylcytosine. Science. 2013;339(6118):448-52.

21. Hajkova $P$, Ancelin $K$, Waldmann $T$, Lacoste N, Lange UC, Cesari F, Lee C, Almouzni G, Schneider R, Surani MA. Chromatin dynamics during epigenetic reprogramming in the mouse germ line. Nature. 2008:452(7189):877-81.

22. Hajkova P, Erhardt S, Lane N, Haaf T, El-Maarri O, Reik W, Walter J, Suran MA. Epigenetic reprogramming in mouse primordial germ cells. Mech Dev. 2002;117(1-2):15-23. 
23. Guibert S, Forne T, Weber M. Global profiling of DNA methylation erasure in mouse primordial germ cells. Genome Res. 2012;22(4):633-41.

24. Kobayashi H, Sakurai T, Miura F, Imai M, Mochiduki K, Yanagisawa E, Sakashita A, Wakai T, Suzuki Y, Ito T, et al. High-resolution DNA methylome analysis of primordial germ cells identifies gender-specific reprogramming in mice. Genome Res. 2013;23(4):616-27.

25. Popp C, Dean W, Feng S, Cokus SJ, Andrews S, Pellegrini M, Jacobsen SE, Reik W. Genome-wide erasure of DNA methylation in mouse primordial germ cells is affected by AID deficiency. Nature. 2010;463(7284):1101-5.

26. Liu S, Brind'Amour J, Karimi MM, Shirane K, Bogutz A, Lefebyre L, Sasaki H, Shinkai Y, Lorinczi MC. Setdb1 is required for germline development and silencing of $\mathrm{H} 3 \mathrm{~K} 9 \mathrm{me} 3$-marked endogenous retroviruses in primordial germ cells. Genes Dev. 2014:28(18):2041-55.

27. Sachs M, Onodera C, Blaschke K, Ebata KT, Song JS, Ramalho-Santos M. Bivalent chromatin marks developmental regulatory genes in the mouse embryonic germline in vivo. Cell Rep. 2013;3(6):1777-84.

28. Lesch BJ, Dokshin GA, Young RA, McCarrey JR, Page DC. A set of genes critical to development is epigenetically poised in mouse germ cells from fetal stages through completion of meiosis. Proc Natl Acad Sci U S A. 2013; 110(40):16061-6.

29. Ng JH, Kumar V, Muratani M, Kraus P, Yeo JC, Yaw LP, Xue K, Lufkin T, Prabhakar $\mathrm{S}, \mathrm{Ng} \mathrm{HH}$. In vivo epigenomic profiling of germ cells reveals germ cell molecular signatures. Dev Cell. 2013;24(3):324-33.

30. Walter M, Teissandier A, Pérez-Palacios R, Bourc'his D. An epigenetic switch ensures transposon repression upon dynamic loss of DNA methylation in embryonic stem cells. eLife. 2016;5:e11418.

31. Luense LJ, Wang X, Schon SB, Weller AH, Lin Shiao E, Bryant JM, Bartolomei MS, Coutifaris C, Garcia BA, Berger SL. Comprehensive analysis of histone post-translational modifications in mouse and human male germ cells. Epigenet Chromatin. 2016;9:24.

32. Mu WP, Starmer J, Shibata Y, Yee D, Magnuson T. EZH1 in germ cells safeguards the function of PRC2 during spermatogenesis. Dev Biol. 2017;424(2):198-207.

33. Brykczynska U, Hisano M, Erkek S, Ramos L, Oakeley EJ, Roloff TC, Beisel C, Schubeler D, Stadler MB, Peters AH. Repressive and active histone methylation mark distinct promoters in human and mouse spermatozoa. Nat Struct Mol Biol. 2010;17(6):679-87.

34. Hammoud SS, Nix DA, Hammoud AO, Gibson M, Cairns BR, Carrell DT. Genome-wide analysis identifies changes in histone retention and epigenetic modifications at developmental and imprinted gene loci in the sperm of infertile men. Hum Reprod. 2011;26(9):2558-69.

35. Hammoud SS, Nix DA, Zhang H, Purwar J, Carrell DT, Cairns BR. Distinctive chromatin in human sperm packages genes for embryo development. Nature. 2009;460(7254):473-8.

36. Erkek S, Hisano M, Liang CY, Gill M, Murr R, Dieker J, Schubeler D, van der Vlag J, Stadler MB, Peters AH. Molecular determinants of nucleosome retention at $\mathrm{CpG}$-rich sequences in mouse spermatozoa. Nat Struct Mol Biol. 2013;20(7):868-75

37. Samans B, Yang Y, Krebs S, Sarode GV, Blum H, Reichenbach M, Wolf E, Steger K, Dansranjavin T, Schagdarsurengin U. Uniformity of nucleosome preservation pattern in mammalian sperm and its connection to repetitive DNA elements. Dev Cell. 2014;30(1):23-35.

38. Royo H, Stadler MB, AHFM P. Alternative computational analysis shows no evidence for nucleosome enrichment at repetitive sequences in mammalian spermatozoa. Dev Cell. 2016;37(1):98-104.

39. Dansranjavin $T$, Schagdarsurengin $U$. The rationale of the inevitable, or why is the consideration of repetitive DNA elements indispensable in studies of sperm nucleosomes. Dev Cell. 2016;37(1):13-4.

40. Prokopuk L, Stringer JM, Hogg K, Elgass KD, Western PS. PRC2 is required for extensive reorganization of $\mathrm{H} 3 \mathrm{~K} 27 \mathrm{me} 3$ during epigenetic reprogramming in mouse fetal germ cells. Epigenet Chromatin. 2017;10:7.

41. Szabo PE, Hubner K, Scholer H, Mann JR. Allele-specific expression of imprinted genes in mouse migratory primordial germ cells. Mech Dev. 2002;115(1-2):157-60.

42. van den Bergen JA, Miles DC, Sinclair AH, Western PS. Normalizing gene expression levels in mouse fetal germ cells. Biol Reprod. 2009;81:362-70.

43. Blewitt M, Whitelaw E. The use of mouse models to study epigenetics. Cold Spring Harb Perspect Biol. 2013;5(11):a017939.

44. Garrick D, Fiering S, Martin DI, Whitelaw E. Repeat-induced gene silencing in mammals. Nat Genet. 1998;18(1):56-9.

45. Robertson G, Garrick D, Wilson M, Martin DI, Whitelaw E. Age-dependent silencing of globin transgenes in the mouse. Nucleic Acids Res. 1996;24(8): $1465-71$.
46. Poliseno L, Marranci A, Pandolfi PP. Pseudogenes in human cancer. Front Med. 2015;2:68.

47. Ciabrelli F, Comoglio F, Fellous S, Bonev B, Ninova M, Szabo Q, Xuereb A, Klopp C, Aravin A, Paro R, et al. Stable Polycomb-dependent transgenerational inheritance of chromatin states in Drosophila. Nat Genet. 2017;49(6):876-86.

48. Gaydos L, Wang W, Strome S. Gene repression. H3K27me and PRC2 transmit a memory of repression across generations and during development. Science. 2014;345(6203):1515-8.

49. Teperek M, Simeone A, Gaggioli V, Miyamoto K, Allen GE, Erkek S, Kwon T, Marcotte EM, Zegerman P, Bradshaw CR, et al. Sperm is epigenetically programmed to regulate gene transcription in embryos. Genome Res. 2016; 26(8):1034-46.

50. Xia B, Gerstin E, Schones DE, Huang W, Steven de Belle J. Transgenerational programming of longevity through E(z)-mediated histone H3K27 trimethylation in Drosophila. Aging. 2016;8(11):2988-3008.

51. Zenk F, Loeser E, Schiavo R, Kilpert F, Bogdanovic O, lovino N. Germ lineinherited H3K27me3 restricts enhancer function during maternal-to-zygotic transition. Science. 2017;357(6347):212-6.

52. Whitelaw NC, Whitelaw E. How lifetimes shape epigenotype within and across generations. Hum Mol Gen. 2006;15(2):R131-7.

53. Lander ES, Consortium IHGS, Linton LM, Birren B, Nusbaum C, Zody MC, Baldwin J, Devon K, Dewar K, Doyle M, et al. Initial sequencing and analysis of the human genome. Nature. 2001;409(6822):860-921.

54. Cordaux R, Batzer MA. The impact of retrotransposons on human genome evolution. Nat Rev Genet. 2009;10(10):691-703.

55. Singer MF. Sines and lines — highly repeated short and long interspersed sequences in mammalian genomes. Cell. 1982;28(3):433-4.

56. Jurka J. Repeats in genomic DNA: mining and meaning. Curr Opin Struct Biol. 1998;8(3):333-7.

57. Zupunski V, Gubensek F, Kordis D. Evolutionary dynamics and evolutionary history in the RTE clade of non-LTR retrotransposons. Mol Biol Evol. 2001; 18(10):1849-63.

58. Ewing $\mathrm{AD}$, Kazazian $\mathrm{HH}$. Whole-genome resequencing allows detection of many rare LINE-1 insertion alleles in humans. Genome Res. 2011;21(6):98590.

59. Poliseno L. Pseudogenes: newly discovered players in human cancer. Sci Signal. 2012;5(242):re5.

60. Daxinger L, Oey H, Isbel L, Whitelaw NC, Youngson NA, Spurling A, Vonk KK, Whitelaw E. Hypomethylation of ERVs in the sperm of mice haploinsufficient for the histone methyltransferase Setdb1 correlates with a paternal effect on phenotype. Sci Rep. 2016;6:25004.

61. Ding WY, Lin L, Cheh B, Dai JW. L1 elements, processed pseudogenes and retrogenes in mammalian genomes. IUBMB Life. 2006;58(12):677-85.

62. Cohen AS, Gibson WT. EED-associated overgrowth in a second male patient. J Hum Genet. 2016:61(9):831-4.

63. Cohen AS, Tuysuz B, Shen Y, Bhalla SK, Jones SJ, Gibson WT. A novel mutation in EED associated with overgrowth. J Hum Genet. 2015;60(6): 339-42.

64. Gibson WT, Hood RL, Zhan SH, Bulman DE, Fejes AP, Moore R, Mungall AJ, Eydoux P, Babul-Hirji R, An J, et al. Mutations in EZH2 cause weaver syndrome. Am J Hum Genet. 2012;90(1):110-8.

65. Tatton-Brown K, Hanks S, Ruark E, Zachariou A, Duarte Sdel V, Ramsay E, Snape K, Murray A, Perdeaux ER, Seal S, et al. Germline mutations in the oncogene EZH2 cause weaver syndrome and increased human height. Oncotarget. 2011;2(12):1127-33.

66. Erhardt S, Su IH, Schneider R, Barton S, Bannister AJ, Perez-Burgos L, Jenuwein T, Kouzarides T, Tarakhovsky A, Surani MA. Consequences of the depletion of zygotic and embryonic enhancer of zeste 2 during preimplantation mouse development. Development. 2003;130(18):4235-48.

67. Boerke A, Dieleman SJ. Gadella BM. A possible role for sperm RNA in early embryo development. Theriogenology. 2007;68:S147-55.

68. Ostermeier GC, Miller D, Huntriss JD, Diamond MP, Krawetz SA. Reproductive biology — delivering spermatozoan RNA to the oocyte. Nature. 2004;429(6988):154.

69. Dalgaard K, Landgraf K, Heyne S, Lempradl A, Longinotto J, Gossens K, Ruf M, Orthofer M, Strogantsev R, Selvaraj M, et al. Trim28 Haploinsufficiency triggers bi-stable epigenetic obesity. Cell. 2016;164(3):353-64.

70. Gardner DK, Lane M. Mammalian preimplantation embryo culture. Methods Mol Biol. 2014;1092:167-82.

71. Borg CL, Wolski KM, Gibbs GM, O'Bryan MK. Phenotyping male infertility in the mouse: how to get the most out of a 'non-performer'. Hum Reprod Update. 2010;16(2):205-24. 
72. Robb GW, Amann RP, Killian GJ. Daily sperm production and epididymal sperm reserves of pubertal and adult rats. J Reprod Fertil. 1978;54(1):103-7.

73. Cotton L, Gibbs GM, Sanchez-Partida LG, Morrison JR, de Kretser DM, O'Bryan MK. FGFR-1 signaling is involved in spermiogenesis and sperm capacitation (vol 119, pg 75, 2006). J Cell Sci. 2006;119(3):604.

74. McFarlane L, Truong V, Palmer JS, Wilhelm D. Novel PCR assay for determining the genetic sex of mice. Sex Dev. 2013;7(4):207-11.

75. Miles DC, van den Bergen JA, Sinclair AH, Western PS. Regulation of the female mouse germ cell cycle during entry into meiosis. Cell Cycle. 2010; 9(2):408-18.

76. Western PS, Miles DC, van den Bergen JA, Burton M, Sinclair AH. Dynamic regulation of mitotic arrest in fetal male germ cells. Stem Cells. 2008;26(2): 339-47.

77. Gustin SE, Hogg K, Stringer JM, Rastetter RH, Pelosi E, Miles DC, Sinclair AH, Wilhelm D, Western PS. WNT/beta-catenin and p27/FOXL2 differentially regulate supporting cell proliferation in the developing ovary. Dev Biol. 2016:412(2):250-60.

78. Gustin SE, Stringer JM, Hogg K, Sinclair AH, Western PS. FGF9, activin and TGFbeta promote testicular characteristics in an XX gonad organ culture model. Reproduction. 2016;152(5):529-43.

79. Bolger AM, Lohse M, Usadel B. Trimmomatic: a flexible trimmer for Illumina sequence data. Bioinformatics. 2014:30(15):2114-20.

80. Trapnell C, Hendrickson DG, Sauvageau M, Goff L, Rinn JL, Pachter L. Differential analysis of gene regulation at transcript resolution with RNA-seq. Nat Biotechnol. 2013;31(1):46-53.

81. Forster SC, Finkel AM, Gould JA, Hertzog PJ. RNA-eXpress annotates novel transcript features in RNA-seq data. Bioinformatics. 2013;29(6):810-2.

82. Law CW, Chen Y, Shi W, Smyth GK. Voom: precision weights unlock linear model analysis tools for RNA-seq read counts. Genome Biol. 2014;15(2):R29.

83. Liberzon A, Subramanian A, Pinchback R, Thorvaldsdottir H, Tamayo P, Mesirov JP. Molecular signatures database (MSigDB) 3.0. Bioinformatics. 2011;27(12):1739-40.

84. Dahl JA, Collas P. MicroChIP. Chromatin immunoprecipitation for small cell numbers. Methods Mol Biol. 2009;567:59-74.

85. Dahl JA, Reiner AH, Collas P. Fast genomic muChIP-chip from 1,000 cells. Genome Biol. 2009;10(2):R13.

86. Boyer LA, Plath K, Zeitlinger J, Brambrink T, Medeiros LA, Lee TI, Levine SS, Wernig M, Tajonar A, Ray MK, et al. Polycomb complexes repress developmental regulators in murine embryonic stem cells. Nature. 2006; 441(7091):349-53.

Ready to submit your research? Choose BMC and benefit from:

- fast, convenient online submission

- thorough peer review by experienced researchers in your field

- rapid publication on acceptance

- support for research data, including large and complex data types

- gold Open Access which fosters wider collaboration and increased citations

- maximum visibility for your research: over $100 \mathrm{M}$ website views per year

At $\mathrm{BMC}$, research is always in progress.

Learn more biomedcentral.com/submissions 